Originalveröffentlichung in: Deuffic, Jean-Luc (Hrsg.): Reliques et sainteté dans l'espace médiéval, SaintDenis 2006, S. 33-63 (Pecia : ressources en médiévistique ; 8/11.2005)

\title{
Philippe Cordez
}

\section{Gestion et médiation des collections de reliques au Moyen Age Le témoignage des authentiques et des inventaires}

\section{Les reliques: entre hagiographie et histoire des collections}

Poussières, liquides, parcelles d'os ou de tissu sont des objets bien discrets ${ }^{1}$. C'est pourtant parmi eux que le Moyen Age a choisi une bonne part de ses objets les plus sacrés, représentant sur terre puissances et mystères célestes. Depuis la Réforme et surtout les Lumières, il apparaît pleinement que l'on a alors cru que presque l'infini se trouvait dans presque rien. Apparaissant aujourd'hui extrême, voire déroutant, le phénomène des reliques est un excellent terrain pour étudier les mécanismes de fabrication des objets sociaux et leurs modalités de fonctionnement. Un objet en soi, chose ou œuvre, ne fait qu'être là, n'a pas d'âme et ne veut rien dire. Toute sa force réside dans cette présence brute et inarticulée. Toujours ambivalentes, matières et formes singulières entrent dès le premier contact sensoriel dans une résonance sourde avec la culture de chacun. L'objet n'acquiert de "sens" propre, c'est-à-dire selon le mot de Krzysztof Pomian sa qualité de "sémiophore"2, qu'appréhendé au sein d'un dispositif matériel et mental particulier, qui déterminera son instrumentalisation sociale.

\footnotetext{
${ }^{1}$ Je remercie Paul BERTRAND pour sa relecture attentive et pour m'avoir communiqué son texte: Authentiques de reliques: authentiques ou reliques?, à paraitre in Le Moyen Age. Nos points de vue sont complémentaires, et nous comptons poursuivre ensemble une étude des documents écrits liés aux reliques.

${ }^{2}$ Cf. en particulier: Pour une histoire des sémiophores. A propos des vases des Médicis, 1986, repris sous le seul sous-titre in Des saintes reliques à l'art moderne. Venise-Chicago. XIII'-XX' siècle, Paris, Gallimard, 2003, p. $147-$ 161.
} 
Les reliques sont souvent le minimum imaginable de l'objet signifiant, leur infime présence au monde rendant quasi impossible leur interprétation pour qui n'est pas averti, en particulier l'homme du XXI ${ }^{\mathrm{e}}$ siècle. Dans son indétermination formelle, la relique est un signifiant quasi nul, donc elle peut exprimer l'infini, signifié quasi pur. Il n'y a de forme et de matière que de quoi fonder la présence de l'absolu, sans que ces contingences, réduites à leur plus simple expression, ne puissent parasiter le message sacré. C'est par un ensemble de médias, matériels, performatifs et textuels, que peut se construire l'extrême sacralité des reliques. Les hommes du Moyen Age mettent ainsi en scène, pour eux-mêmes, une réification des personnes divines qu'ils considèrent comme des sujets doués de volonté protégeant leurs communautés. Dans cette opération, les sujets déclarés saints deviennent des objets sacrés, reliques qui sont manipulées et mobilisées comme enjeux sociaux. Au résultat, un pouvoir surnaturel est institué par les hommes dans un objet inanimé, lequel sera présenté et reconnu par eux comme d'origine divine, une inversion qui définit la catégorie même d'objet sacré et son rôle dans la société.

Intermédiaires entre les reliques et les hommes, les multiples médias qui les entouraient et les faisaient exister livrent aujourd'hui à l'historien ses matériaux de base. Leurs contenants, les reliquaires et les images via lesquels les reliques étaient données à voir et à toucher, en sont le type le plus spectaculaire. A mesure que l'efficacité sociale des reliques était remise en cause et dépérissait, ces objets ont acquis une valeur propre, rempli les musées et suscité l'attention des historiens de l'art. Des recherches récentes visent à les

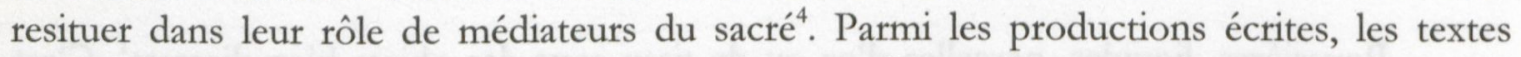
hagiographiques précisaient l'identité des saints, tandis que les récits de translations et de miracles prouvaient l'authenticité des reliques et démontraient leurs pouvoirs. Les textes liturgiques témoignent des rituels où elles étaient impliquées. Mais les écrits les plus massivement répandus et peut-être les plus fondamentaux sont sans doute les authentiques et les inventaires.

Contiguës aux reliques, les "authentiques" prouvent la présence matérielle des saints, en jouant de l'écriture comme médiatrice du sacré sur terre. Mais justement, le terme est problématique: il n'est pas médiéval, semble n'apparaître qu'à l'époque moderne et traduit sans doute une conception juridique de l'authenticité qui est étrangère au Moyen Age. Si l'on veut étudier la genèse de cette conception, on est amené à élargir la notion d'authentique de relique à "tout type de document identifiant et/ou authentifiant une relique de saint et que l'on peut retrouver dans une châsse”. C'est ce qu'a proposé N.

\footnotetext{
${ }^{3}$ Sur les objets sacrés cf. Maurice GODELIER, L'énigme du don, Paris, Fayard, 1996, p. 167-172 et 244-246.

${ }^{4}$ Cf. en particulier les recherches menées depuis 2002 à l'université de Hambourg par Bruno REUDENBACH et Gia TOUSSAINT sous le titre "Reliquiare als Wahrnehmung und Konstruktion von Heiligkeit". Cf. Leur article Die Wahrnehmung und Deutung von Heiligen. Überlegungen zur Medialität von Reliquiaren, in Das Mittelalter, 8, 2003, p. 34-40, et les actes du colloque Reliquiare im Mittelalter. Kunst, Kult, Kontext, Berlin, Akademie Verlag, à paraître.
} 
Herrmann-Mascard dans son étude de la "formation coutumière d'un droit", et à sa suite P. Bertrand, qui renouvelle cette perspective sous l'angle d'une histoire de l'écriture et de la preuve $^{5}$. On considère ici ces documents sous un autre angle, à savoir en tant qu'acteurs et témoins concrets de la gestion et de la médiation de séries de reliques. De manière assez habituelle, mais surtout très pragmatique pour notre propos, on limitera donc la notion d'authentique à la première strate d'écriture, c'est-à-dire les étiquettes physiquement liées aux reliques qui sans elles se perdraient, à l'exclusion d'autres documents qui identifient ou authentifient. On désignera par "inventaire de reliques" toute série de noms de reliques, que le document se trouve auprès des reliques ou non. Ces acceptions sont provisoires, dans l'attente d'une terminologie plus fine qui rende mieux compte des types variés de documents et des problèmes qu'ils posent.

Il y a de toute évidence un déficit d'intérêt des historiens des collections pour les reliques. Sans doute est-ce parce que malgré leur importance au cours du long Moyen Age et même si elles sont conservées, l'attrait qu'elles exerçaient jadis s'est déplacé, notamment vers les grands objets d'art ou de savoir. Les rares études du collectionnisme médiéval le réduisent à la renaissance d'un marché de l'art, qui rappelle le modèle antique, et ignorent les reliques ${ }^{6}$. Or les collections de reliques me semblent justement constituer par excellence les collections médiévales, en tant qu'ensembles d'objets réunis volontairement selon un système où ils s'ordonnent. Certes, d'autres types d'objets de pouvoir font système au Moyen Age, forment des séries, sont inventoriés. Ainsi les insignes des clercs et de certains laïcs, ou les instruments et ornements liturgiques liés à un autel. Mais ils ne sont pas rassemblés librement, car leur usage prévaut toujours sur leur réunion. Objets de don et archives, quant à eux, sont conservés pour leur valeur mémorielle et légale, sans être collectés pour eux mêmes. Seuls certains livres sont vraiment collectionnés, sur le critère de l'auteur du texte, personnalisation qui les rapproche des reliques, comme plus tard les œuvres d'artistes ${ }^{7}$. On voit que les accumulations médiévales reposent sur des principes beaucoup plus hétérogènes que les collections modernes et contemporaines, qui réunissent les productions de la nature et des artistes et ont perdu, en apparence, tout caractère rituel et sacré. Une étude des collections de reliques devrait pourtant éclairer leurs racines.

\footnotetext{
${ }^{5}$ Cf. Nicole HerrmanN-MASCARD, Les reliques des saints: formation coutumière d'un droit, Paris, Klincksieck, 1975 et BERTRAND, Authentiques de reliques, dont je tire la citation.

${ }^{6}$ Krzysztof POMIAN, Collezionismo, in Enciclopedia dell'arte medievale, Rome, Istituto della Enciclopedia Italiana, t. 5, 1994, 156-160. Même dans son livre intitulé Des saintes reliques à l'art moderne, l'auteur ne consacre que quelques pages aux reliques, exclusivement vénitiennes, qui servent surtout de point de départ à sa démonstration sans être déterminantes.

7 Tous ces objets, les monnaies également, se placent sous l'image générale du trésor, indivisible et infini, plutôt que sous celle de la collection. Ces pratiques et discours font l'objet de ma thèse sur Les "trésors d'église" au Moyen Age.
} 
La possibilité même de la collection des reliques dépend étroitement de la matérialité de ces dernières, c'est-à-dire des conditions de leur production, à partir de l'officialisation du christianisme. Les plus simples des collections furent réunies par les pèlerins de terre sainte : le récit biblique en fournit la trame. Dans un processus de concrétisation de l'imaginaire, les pèlerins "inventent" ces objets et condensent la "topographie légendaire des évangiles" en une série cohérente d'échantillons minéraux ${ }^{8}$. D’autre part, le culte porté aux saints donnait lieu lui aussi à la production de reliques, le recours aux restes corporels permettant d'instrumentaliser la part d'objet présente dans tout sujet. A la suite de substances diverses ayant touché les tombeaux, corps et fragments de corps se mirent à circuler, à mesure que l'interdiction romaine d'ouvrir les tombes était levée. Apparue dès le $\mathrm{IV}^{\mathrm{e}}$ siècle en Orient, la fragmentation s'établit plus lentement en Occident, où elle ne semble s'être imposée qu’à partir du VII ${ }^{\mathrm{e}}$ ou du VIII ${ }^{\mathrm{e}}$ siècle, ou plus tard selon les contextes?

Le $\mathrm{VI}^{\mathrm{e}}$ siècle paraît avoir connu une augmentation notable des transactions de reliques de toutes sortes, attestée à la fois par des textes - Grégoire le Grand, Grégoire de Tours - et sur le plan archéologique par les plus anciennes authentiques de reliques conservées. A côté des reliques locales que tous les fidèles s'approprient, des reliques plus prestigieuses, romaines ou orientales, deviennent des signes de distinction que s'échangent les puissants. A la même époque et de manière corrélative apparaissent les premières entreprises systématiques d'accumulations de reliques, c'est-à-dire les premières figures de collectionneurs. Certains ensembles durent être transmis par don ou héritage, tandis que d'autres aboutissaient dans des églises ${ }^{10}$ : églises fondées par les donateurs, ou églises puissantes auxquelles ceux-ci souhaitaient s'affilier. Les reliques entraient alors dans des institutions voulues pérennes, qui furent les seules à même de conserver, dans certains cas, des authentiques de reliques jusqu'à nos jours: c'est le temps long des sanctuaires d'Europe. Circulation et accumulation des reliques s'intensifièrent avec le temps. Certaines transactions donnèrent lieu à la rédaction de récits de translation, dont la grande période court du

\footnotetext{
${ }^{8}$ Cf. Yitzhak Hen, Les authentiques des reliques de la Terre Sainte en Gaule franque, in Le Moyen Age. Revue d'bistoire et de philologie, 105, 1999, p. 71-90; Cynthia HAHN, Loca Sancta Souvenirs: Sealing the Pilgrim's Experience, in Robert OUSTERHOUT (éd.), The Blessings of Pilgrimage, Urbana, University of Illinois, 1990, p. 8595; Maurice Halbwachs, La Topographie légendaire des Evangiles en Terre Sainte, Paris, Presses universitaires de France, 1941.

${ }^{9}$ Cf. Luigi CANETTI, Reliquie, martirio e anatomia: culto dei santi e pratiche dissettorie fra tarda Antichità e primo Medioevo, in Le cadavre. Anthropologie, archéologie, imaginaire social (Moyen Age, Renaissance), Turnhout: Brepols, 1999 (Micrologus, VII), p. 113-153; l'hostilité à la fragmentation est étudiée par Arnold ANGENENDT, Der "ganze" und "unverweste" Leib - eine Leitidee der Reliquienverehrung bei Gregor von Tours und Beda Venerabilis, in Hubert MORDEK (éd.), Aus Archiven und Bibliotheken. Festschrift für Raymund Kottje zum 65. Geburtstag, Francfort sur le Main/etc., Lang, 1992 (Freiburger Beiträge zur mittelalterlichen Geschichte 3), p. 33-50.

${ }^{10}$ Cf. Jas ELSNER, Replicating Palestine and reversing the reformation. Pilgrimage and collecting at Bobbio, Monza and Walsingham, in Journal of the History of Collections, 9/1, 1997, p. 117-130; Cynthia HAHN, Early Medieval Reliquary Collections, in REUDENBACH, TOUSSAINT (éd.), Reliquiare.
} 
VIII $^{\mathrm{e}}$ au $\mathrm{X}^{\mathrm{e}}$ siècle $^{11}$. D'autres ont laissé moins de traces. La construction de la valeur d'une relique est chaque fois une histoire singulière, politique et sociale ${ }^{12}$ : la rédaction d'un récit n'en est qu'un élément possible, et l'intensification de la circulation des reliques finira par en réduire l'utilité.

De manière plus brève, les acquisitions de reliques furent aussi notées dans des textes historiographiques consacrés à un lieu ou à un prélat, ce qui permet parfois de repérer des figures de collectionneurs ${ }^{13}$. Mais là encore ces sources mettent l'accent sur les acquisitions singulières, invitant à les considérer sur le mode biographique: biographies des acteurs de la translation et du collectionneur, des saints selon l'approche hagiographique traditionnelle, ou encore la biographie culturelle des reliques elles-mêmes, pour reprendre une notion d'anthropologie économique désignant le phénomène de construction de la valeur des biens $^{14}$. S'en tenir là, c'est d'une part négliger la masse des transactions les plus discrètes, et d'autre part devoir ignorer ce que les reliques devinrent en pratique dans les institutions, une fois passées l'acquisition et son interprétation, en dehors des reliques les plus valorisées. D'où la nécessité d'étudier pour elles mêmes les accumulations de reliques formées progressivement dans les sanctuaires.

Une collection de reliques est d'abord une collection d'authentiques. Mises à part des publications isolées, seules les plus anciennes d'entre elles ont été étudiées, en particulier sous l'aspect paléographique. On sait ainsi que les sanctuaires de l'actuel territoire français en ont conservé 267 antérieures à l'an 800, dont deux ensembles importants: 139 authentiques au monastère de Chelles, 94 à la cathédrale de Sens ${ }^{15}$. La diversité des écritures y indique des dons étalés dans le temps. Mais les authentiques circulent justement trop facilement pour permettre une étude fiable des collections, qui ne pourrait reposer sur l'analyse d'objets singuliers. Dès l'origine et aussi pour l'historien, les collections ne peuvent

${ }^{11}$ Martin HeinzelmanN, Translationsberichte und andere Quellen des Reliquienkultes, (Turnhout, Brepols, 1979 (Typologie des Sources du Moyen Âge, 33).

${ }^{12}$ Comme le souligne Hedwig RÖCKELEIN, Reliquientranslationen nach Sachsen. Über Kommunikation, Mobilität und Öffentlichkeit in Frübmittelalter, Sttugart, Thorbecke, 2002 (Beihefte der Francia, 48).

${ }^{13} \mathrm{Cf}$. ci-dessous pour Angilbert de Saint-Riquier. A propos de plusieurs prélats lotharingiens: Anne WAGNER,

Collection de reliques et pouvoir épiscopal au Xe siècle. L'exemple de l'évêque Thierry $\mathrm{I}^{\mathrm{er}} \mathrm{de}$ Metz, in Revue d'Histoire de l'Eglise de France, 83, 1997, p. 317-341.

${ }^{14}$ Cf. Igor KOPYTOFF, The Cultural Biography of Things: Commoditization as Process, in Arjun APPADURAI (éd), The Social Life of Things: Commodities in Cultural Perspective, Cambridge, Cambridge University Press, 1986 (Cambridge Studies in Social \& Cultural Anthropology), p. 64-91. La notion a été appliquée au problème des translations de reliques par RÖCKELEIN, Reliquientranslationen, p. 149-154.

${ }^{15}$ Tous ces documents ont été édités et publiés en facsimilé: Chartae latinae antiquiores. Facsimile-Edition of the Latin Charters prior to the Ninth Century, t. 18, France VI, éd. Hartmut ATSMA, Pierre GASNAULT, Robert MARICHAL, Jean VEZIN, Dietikon/Zurich, Graf, 1985 et t. 19, France VII, éd. Hartmut ATSMA, Robert MARICHAL, Jean VEZIN, Dietiekon/Zurich, Graf, 1987. Je n'ai pu consulter Bruno GALLAND, Les authentiques de reliques du sancta sanctorum, Città del Vaticano, Studi e testi, 2004. 
être appréhendées qu'à travers les inventaires, qui les constituent comme telles, ordonnent les ensembles et en présentent une vision globale. Or les inventaires de reliques sont à peine mieux édités et commentés que les authentiques ${ }^{16}$. Ils ont généralement été interprétés selon le modèle biographique, en cherchant à reconstituer les translations successives et à travers elles l'histoire des lieux et des réseaux ${ }^{17}$, ou bien sur un mode quantitatif qui pose problème ${ }^{18}$.

Un outil de travail a été constitué, à partir d'éditions souvent dispersées au sein des historiographies francophone et germanophone, par un premier rassemblement de documents. On l'a provisoirement interrompu lors du constat que les éléments nouveaux s'inscrivaient en gros dans les catégories définies. Ceci permet dès maintenant d'éclairer plusieurs points cruciaux d'une histoire des collections de reliques, dont le présent essai voudrait être un premier jalon en attendant un dépouillement plus poussé.

\section{La rédaction des inventaires: techniques et politiques de gestion}

Si l'acte de rédaction d'un inventaire peut être défini comme un moment fondateur dans la constitution d'une collection, alors on voudrait connaitre les circonstances concrètes, les conditions techniques présidant à la naissance de ces textes. Deux types

16 La plus importante étude de documents liés aux reliques est à ma connaissance Ernst Alfred STÜCKELBERG, Geschichte der Reliquien in der Schweiz, 2 t., Zurich, 1902-1908 (Verlag der Schweizerischen Gesellschaft für Volkskunde, 1 et 5). Près de 3000 documents du IVe siècle à 1907, dont ca. 600 antérieurs à 1500 , y sont présentés chronologiquement dans des notices renvoyant au document ou plus souvent à des publications diverses, avec un index des lieux. L'analyse typologique se limite à un long chapitre de l'introduction ("sources", t. 1 p. XI-LXXII, p. XXXIV-LIII sur les inventaires). Aller au-delà supposerait de dépouiller le corpus, ce que l'imprécision fréquente quant aux supports matériels des inventaires et l'ancienneté des références rend difficile. Signalons aussi l'édition par Philippe GEORGE, depuis le début des années 1980, de documents de l'ancien diocèse de Liège: $\mathrm{cf}$. la liste de ses travaux dans son Reliques et arts précieux en pays mosan. Du baut Moyen Age à l'époque contemporaine, Liège, éd. du Céfal, 2002, par ailleurs un livre de synthèse sans appareil scientifique.

${ }^{17} \mathrm{La}$ littérature francophone cite souvent pour exemple d'édition et d'étude Dom Jacques DuBOIS, Le trésor des reliques de l'abbaye du Mont-Saint-Michel, in Millénaire monastique du Mont-Saint-Michel, 1, Paris, P. Lethielleux, 1967, p. 501-593; repris in ID., Aspects de la vie monastique en France au Moyen Age, Londres, 1993 (j'utilise plus loin l'édition de 1967); complété par Reliques apportées de Pouilles au Mont-Saint-Michel au XIe siècle, in Bulletin des Amis du Mont-Saint-Michel, 101, 1996, p. 49-51.

18 François Neveux, Les reliques du Mont-Saint-Michel, in Pierre Bouet, Giorgio OTranto, André VAUCHEZ (dir.), Culte et pèlerinages à Saint Michel en Occident. Les trois monts dédiés à l'Archange, Rome, 2003 (Collection de l'École française de Rome, 316), p. 245-269. Après avoir éliminé d'autres listes plus courtes, l'auteur a rassemblé huit inventaires d'origines diverses datant du XII ${ }^{e}$ au XVII ${ }^{e}$ siècles. Les tenant d'emblée pour des inventaires complets de collections médiévales, ignorant qu'une église n'accordait pas la même attention à toutes ses reliques, il établit et compare des pourcentages de types de saints (martyrs, papes, bretons...) pour en tirer des conclusions sur leur culte. 
d'inventaires peuvent structurellement être distingués: les inventaires de reliques enfermées dans des autels, et les inventaires de reliques mobiles, conservées dans toutes sortes de reliquaires. Le recours à l'un ou l'autre type de texte s'inscrit chaque fois dans une situation différente, qu'il faut toujours interpréter, ce qu'on verra à travers quelques exemples.

\section{Les notices de consécration d'autels}

Parmi les lieux d'accueil des reliques, l'autel tient une place particulière. Le dépôt de reliques dans les autels, par lequel on associe les saints à la commémoration de l'eucharistie, remonte dans son principe au $\mathrm{IV}^{\mathrm{e}}$ siècle. La pratique semble avoir été fréquente dès le $\mathrm{VI}^{\mathrm{e}}$ siècle et devint ensuite systématique, sans pour autant être obligatoire. Vers le VI ${ }^{\mathrm{e}}$ siècle aussi, la multiplication du nombre des autels dans les églises implique déjà de disposer de séries de reliques. On passa également du dépôt d'une seule relique par autel à celui d'une série de reliques. Il parut nécessaire d'établir la liste des reliques déposées, ce que prescrivirent bientôt les textes normatifs correspondants, c'est-à-dire les rituels de consécration des autels.

Mis au point vers le milieu du $\mathrm{X}^{\mathrm{e}}$ siècle à Mayence, le Pontifical romano-germanique est une compilation de rituels à l'usage des évêques qui allait rapidement, portée par l'entreprise ottonienne de restauration de l'Empire, être très largement diffusée. Il y est indiqué, et cela est alors nouveau dans les rituels, que l'évêque consacrant un autel doit “annoncer et au clergé et au peuple" la liste des reliques qu'il y déposera ${ }^{19}$. Ce type de texte existait déjà depuis un certain temps, comme en témoigne un morceau de parchemin où l'on lit la notice de consécration d'une église de Melun en 809 avec une liste de 27 reliques $^{20}$. Il faut attendre le $\mathrm{XI}^{\mathrm{e}}$ siècle pour trouver dans un cérémonial isolé, celui de Vich, l'exigence du dépôt d'une telle carta par le consécrateur de l'autel, précisant la date, son nom et celui des reliques ${ }^{21}$.

\footnotetext{
${ }^{19}$ Le pontifical romano-germanique du dixième siècle, éd. C. VOGEL, R. ELZE, Rome, Città del Vaticano, 1964 (Studi et Testi, 227), Ordo XL, c. 128, p. 169: His ita per ordinem gestis, cum redierit iterum ad ostium aecclesiae, facto silentio, babeat pontifex verbum ad plebem [...] et annuntiet tam clero quam populo, in cuius honore constructa et dedicata sit aecclesia vel etiam nomina sanctorum ibi quiescentium. Ce texte est répété avec des variantes mineures dans Le pontifical de la curie romaine au XIIIe siècle, éd. Michel ANDRIEU, Le pontifical romain au Moyen Age, 4 t., Rome, Città del Vaticano, 1938-1941 (Studi et Testi, 86-88 et 99), ici t. 2, p. 433.

${ }^{20} \mathrm{La}$ feuille (ht. $23,5 \mathrm{~cm}, 1.7,2 \mathrm{~cm}$ ) se trouve à la cathédrale de Sens. Etait-elle enfermée à l'origine dans un autel, qu'elle aurait quitté dès le Moyen Age? Ed. Maurice PrOU, Eugène ChARTraIre, Authentiques de reliques conservées au Trésor de la cathédrale de Sens, in Mémoires de la Société nationale des Antiquaires de France, 59,1890 , p. $129-172, n^{\circ} 160$, cf. aussi p. 132. Autre exemple à Beromünster en Suisse, une bande de parchemin datée vers la seconde moitié du IX siècle: Iso MÜLLER, Zum mittelalterlichen Reliquiens-chatze von Beromünster, in Der Geschichtsfreund. Mitteilungen des Historischen Vereins der Fünf Orte Luzern, Uni, Schnyv Unterwalden ob und nid dem Wald und Zug, 120,1967, p. 5-40, ici p. 12-17.

${ }^{21}$ Ponat etiam cartam continentam $X$ praecepta legis et principia IV evangeliorum et nomen suum et diem et annum et nomina sanctorum, quorum reliquias reconduntur. Manuscrit conservé au Musée diocésain de Vich, cité par Joseph BRAUN,
} 
Très largement utilisé au XIII ${ }^{\mathrm{e}}$ siècle, le pontifical établi au XII ${ }^{\mathrm{e}}$ siècle à la curie romaine ne prescrit pas cela mais suppose, auprès des reliques que l'évêque consécrateur prélève pour les apporter dans la nouvelle église, la présence d'écritures à regarder diligenter, sans doute des authentiques ${ }^{22}$. L'évêque de Mende Guillaume Durand reprend à la fin du XIII siècle dans son Pontifical, qui supplantera peu à peu celui de Rome, l'injonction de publication orale des listes de reliques à déposer ${ }^{23}$. Surtout, il précise qu'à la veille de la consécration, l'évêque doit placer une "petite charte de parchemin, écrite en grosses lettres" indiquant l'identité des reliques dans le vase contenant les reliques à déposer dans l'autel ${ }^{24}$. Cette charte précisera les noms des saints à qui l'église et l'autel sont dédiés, celui du consécrateur scellant le vasculum des reliques, la date de la consécration, et l'indulgence délivrée aux jours anniversaires de la consécration. On retrouve tout cela dans des pontificaux du $\mathrm{Xv}^{\mathrm{e}}$ siècle $^{25}$. Et de fait, on a parfois retrouvé de telles notices dans des autels. Celle d'un autel de la cathédrale de Gérone, consacré en 1341 aux 11000 vierges et ouvert en 1826, mentionne sept reliques ${ }^{26}$.

Les inventaires compris dans les notices de consécration, dont on verra qu'elles pouvaient être copiées sur divers supports, permettaient de garder la mémoire des reliques enfermées dans les autels. Elles y restaient jusqu'à l'ouverture, qui désaffectait l'autel, rendant nécessaire une nouvelle consécration et donc la rédaction d'un nouveau document. $\mathrm{Si}$ on pouvait oublier l'identité des reliques enfermées dans un autel, cela ne durait que

Der christliche Altar in seiner geschichtlichen Entwicklung, 2 t., Munich, Alte Meister Guenther Koch \& Co., 1924, ici t. 1 , p. 632 n. 2.

22 Primitus decet episcopum cum clericis ire ad antiquam ecclesiam, vel ad locum in qui reliquiae sunt, et ibi diligenter visis reliquiis omnibus et singulatim nominibus scriptis, recondat eas episcopus in aliquo vase sigillo signatas et sic deferant ad tentorium, vel ad locum in quo vigilandae sunt... Le pontifical romain du XII siècle, ordo XVII c. 1, éd. ANDRIEU in Le pontifical romain, t. 1, p. 176. Ce texte prescrit l'annonce publique de la liste, comme le pontifical du $\mathrm{X}^{\mathrm{e}}$ siècle.

${ }^{23}$ Interim autem dum premissa preparantur, pontifex debet annuntiare populo in cuius sancti bonorem et nomen ecclesia est dedicanda et quorum sanctorum reliquiae sunt in altari recondende. Le pontifical de Guillaume Durand, livre II ordo II c. 10, éd. ibid., t. 3 p. 457.

${ }^{24}$ Ibid., p. 456: Ponat etiam ibi carticulam de corio, scriptam de grossa littera, continentem cujusmodi reliquie sunt ibi incluse, et in cujus sancti honorem et nomen ecclesia ipsa et altare dedicantur, nomen consecratoris et indulgentiam quam in anniversario dedicationis die concedit, annum quoque, mensem et diem quibus dedicatur, sigillans ipsum vasculum diligenter...

${ }^{25}$ Cf. BRAUn, Der christliche Altar, t. 1, p. 632, qui considère toutefois que cette prescription serait rare. Il cite le formulaire d'une telle notice dans un pontifical de Bergame, vers 1451 (Vat. lat. 1145): Ponatur ibi etiam cartula, super qua scripta haec verba de grossis litteris: Reverendissimus in Christo pater et dominus dominus N., Dei et apostolicae sedis gratia episcopus talis ecclesiae, consecravit banc ecclesiam sub honore sancti $N$. martyris vel confessoris et hoc altare sub honore sancti $N$. et posuit in eo reliquias sanctorum talis et talis et concessit singulis annis in anniversario dedicationis talem indulgentiam omnibus ipsam ecclesiam visitantibus in forma ecclesiae consueta. Anno a Nativitate Domini nostri Jesu Christi $1451, \ldots$ die... mensis, pontificatus sanctissimi et beatissimi in Christi patris ac domini N. divina providentia papae anno...

${ }^{26}$ Cf. BRAun, Der christliche Altar, t. 1, p. 632. 
jusqu’à son ouverture et la redécouverte de la notice. Le mélange et la confusion des reliques, par contre, étaient impossibles.

La rigueur et la solennité avec lesquelles ont tenait le compte des reliques enfermées dans les autels semble avoir valu également pour certains reliquaires. Certains procèsverbaux de translation paraissent ainsi calqués sur les notices de consécration d'autels, avec date, auteur de la translation et identité des reliques. L'un d'eux, intitulé Titulus capitis beati Alexandri, rappelle qu'à Pâques 1145, l'abbé Wibald de Stavelot plaça dans le chef-reliquaire du pape Alexandre deux reliques de ce saint et d'autres "trouvées avec elles": cinq reliques du Christ, celles des saints Pierre, Agapet et Crispin, de la légion thébaine et des 11000 vierges. Mais c'est une copie du XIII ${ }^{\mathrm{e}}$ siècle de ce procès-verbal qui se trouve aujourd'hui dans le reliquaire ${ }^{27}$, ce qui montre que celui-ci a été ouvert, sans que le texte n'évoque cette ouverture. De quand date réellement l'inventaire que l'auteur du XIII ${ }^{\mathrm{e}}$ siècle attribue à 1145 et à l'abbé Wibald, figure prestigieuse de l'histoire de Stavelot?

\section{Le dépouillement des authentiques}

Une manière certaine d'empêcher toute manipulation de reliques est d'en faire figurer la liste sur le reliquaire lui-même. Sur le revers de trois reliquaires de laiton du milieu du $\mathrm{XIII}^{\mathrm{e}}$ siècle, provenant de l'abbaye cistercienne de Grandselve (Tarn-et-Garonne), sont ainsi gravées des listes particulièrement longues de 32, 34 et 35 reliques. A l'ouverture en 1895 du reliquaire dit de la vraie Croix, on découvrit de petits paquets de soie rouge et jaune auxquels étaient attachées 31 bandes de parchemin, où les noms de chaque relique avaient été inscrits au XIII ${ }^{\mathrm{e}}$ siècle à l'encre noire. Toutes sauf une sont de la même main. Seules trois authentiques manquaient par rapport aux 34 noms de l'inventaire ${ }^{28}$ : après six siècles, le contenu correspondait encore presque à la description.

Ces inscriptions sur des reliquaires ne sont cependant par la règle. Comment avoir une vue d'ensemble quand les reliques sont déposées dans des boîtes ou châsses ouvertes et refermées à loisir et s'en échappent si facilement, ou au contraire quand on oublie après plusieurs décennies ou siècles le contenu d'un reliquaire trop bien clos? Il fallait alors ouvrir

\footnotetext{
${ }^{27}$ Le reliquaire est aux Musées Royaux d'Art et d'Histoire de Bruxelles. Ed. Philippe GEORGE, Documents inédits sur le Trésor des reliques de l'abbaye de Stavelot-Malmedy et dépendances (IXe et XVIII ${ }^{\mathrm{e}}$ siècles), in Académie royale de Belgique. Bulletin de la Commission royale d'bistoire, 153, 1987, p. 65-108 et 129-179; repris in ID., Les reliques de Stavelot-Malmedy. Nouveaux documents, Malmédy, Art et Histoire, 1989, $\mathrm{n}^{\circ} 43$.

${ }^{28}$ Les reliquaires sont aujourd'hui conservés à l'église paroissiale de Bouillac (Tarn-et-Garonne). Authentiques et inscriptions ont été publiées par F. POTTIER, Les authentiques de reliques, in Bulletin de la Société Archéologique de Tarn-et-Garonne, 1912, p. 145-159, ici p. 150 et 152; les inscriptions à nouveau in Robert FAVREAU, Jean Michaud, Bernadette LePLANT-MORA, Ariège, Haute-Garonne, Hautes-Pyrénées, Tarn-et-Garonne, Paris, 1983 (Corpus des inscriptions de la France médiévale, 8), p. 117-124.
} 
les reliquaires, retrouver les authentiques, les lire et les compiler. L'opération n'est pas toujours facile et tel inventaire de Brunswick, en 1482, prend soin d'expliquer le mécanisme d'ouverture d'une certaine boîte d'ivoire ${ }^{29}$. Si les authentiques jadis utilisées pour un inventaire sont conservées, on peut se placer derrière l'épaule du rédacteur et le voir à l'œuvre, observant ses méthodes et éprouvant avec lui ses difficultés. Le 26 mai 1185, l'abbé Erlebald de Stavelot-Malmedy (1158-1192) ouvrit la châsse de saint Simètre de l'église paroissiale de Lierneux, filiale de son abbaye dans la province de Liège. On écrivit un inventaire sur un morceau de parchemin. Celui-ci resta jusqu'à aujourd'hui, avec une série d'authentiques, dans la châsse refaite elle-même au XIII ${ }^{\mathrm{e}}$ siècle $^{30}$ (fig. I/1). Sur les 38 reliques de l'inventaire, 32 sont citées dans 20 des 26 authentiques datant des $\mathrm{IX}^{\mathrm{e}}-\mathrm{X}^{\mathrm{e}}$ au XII siècle ${ }^{31}$. Aucun ordre ne semble avoir gouverné la copie des authentiques, si ce n'est peutêtre que Simètre ouvre la liste juste après Marie et Pierre. Deux reliques mentionnées sur l'une des authentiques, transférées ailleurs quarante ans plus tôt, n'ont logiquement pas été reprises dans l'inventaire ${ }^{32}$. L'interprétation laisse cependant quelques questions ouvertes. Deux ou trois authentiques similaires correspondent parfois à une seule relique de l'inventaire $^{33}$, qui ignore par ailleurs six authentiques ${ }^{34}$, dont trois sont pourtant de la même main que d'autres qui furent utilisées ${ }^{35}$. Si de nouvelles authentiques n'ont pas été glissées depuis dans la châsse, il faut croire que certaines informations ont été synthétisées et d'autres ignorées: soit qu'on n'ait pu lire les authentiques, ce que des altérations ${ }^{36}$ rendent plausible, soient qu'elles aient échappé aux inspecteurs de 1185, qui comme ceux des années 1980 durent les débusquer dans les multiples bourses de tissu où elles étaient disséminées ${ }^{37}$. Quoi qu'il en soit l'inventaire reconnaît ses limites, citant pour finir multe alie reliquie quorum nomina Deus solus novit.

\footnotetext{
29 Item circa seram extrabatur clavus et ibi invenitur instrumentum quod extrabi oportet antequam aperiatur. Andrea BOOCKMANn, Die verlorenen Teile des "Welfenschatzes". Eine Übersicht anhand des Reliquienverzeichnisses von 1482 der Stiftskirche St. Blasius in Braunschweig, Göttingen, Vandenhoeck \& Ruprecht, 1997, p. 133, $\mathrm{n}^{\circ} 17$.

${ }^{30}$ L'inventaire est copié sur la moitié d'un morceau de parchemin plié en deux de 12,3 sur $16,2 \mathrm{~cm}$. Il se termine par Idem abbas petitione fidelium ecclesie, Harduini videlicet inuestiti ecclesie de Lernau sarcofagum in quo reliquie be continebantur; idem abbas apperuit et has predictas reliquias invenit. Ed. avec les autres authentiques par Ph. GEORGE, Les reliques, $\mathrm{n}^{\circ} 1-27$.

${ }^{31}$ Une $33^{e}$ relique apparaît sur l'authentique $\mathrm{n}^{\circ} 28$, la seule moderne, probablement remplacée au XVII ${ }^{e}$ ou au XVIII ${ }^{\mathrm{e}}$ siècle.

32 Authentique $n^{\circ} 26$ : ce sont les reliques du pape Alexandre, transférées dans son chef reliquaire dont il était question plus haut.

${ }^{33}$ Authentiques $\mathrm{n}^{\circ} 1$ et $9 ; 4$ et $21 ; 6,13$ et $25 ; 10,17$ et 26 .

${ }^{34} \mathrm{~N}^{\circ} 2-3,5,12,15,19,23$.

${ }^{35} \mathrm{~N}^{\circ} 2$ à 7 , toutes des reliques de Terre Sainte.

${ }^{36} \mathrm{~N}^{\circ} 3$ et 12 . (une authentique du XVIII' siècle supplée à l'altération)

${ }^{37}$ Ibid., p. 14.
} 
Parmi les reliquaires du Mont-Saint-Michel inventoriés en 1396, il y avait un "petit vase quadrangulaire" dans lequel on trouva des authentiques ${ }^{38}$ dont six sont conservées. Datant probablement du XIII ${ }^{\mathrm{e}}$ siècle, elles recensent 22 des 24 reliques citées dans l'inventaire. La comparaison révèle que l'auteur a recopié ces textes en allant du plus long au plus court - il n'y a pas de mauvaise méthode! Les deux premières authentiques concernent des ensembles homogènes, venus de Terre Sainte et de Saint-Denis. Les deux listes suivantes n'ont pas cette cohérence et reflètent sans doute des rassemblements secondaires. Dans un cas ce sont trois saints normands, le Christ, la Vierge et Marguerite, soit des reliques proches et lointaines qui furent sans doute réunies en Normandie. Comme sur l'authentique des reliques de Terre Sainte, le mot continentur montre que le contenu d'un premier reliquaire a été transféré dans celui qui fut inventorié en 1396. Dans l'édition de 1967, le passage concernant ce reliquaire est articulé par quatre retours à la ligne, qui suivent sans doute la disposition de la copie du $\mathrm{XV}^{\mathrm{e}}$ siècle. La mise en page des listes, ailleurs les item suivis d'un groupe de reliques, peuvent ainsi correspondre à des authentiques de reliques d'origines distinctes: il faut donc accorder la plus grande importance à ces détails pour reconstituer l'histoire d'une collection ${ }^{39}$.

L'auteur de l'inventaire des reliques du Mont-Saint-Michel avoue par quatre fois n'avoir pu utiliser des authentiques trop vieilles (propter vetustatem), noircies (perobscure traditur) ou encore perdues ${ }^{40}$, et mentionne deux fois des reliques anonymes ${ }^{41}$. C'était de fait un exercice toujours difficile que de lire les documents que l'on exhumait. Les auteurs mentionnent assez souvent leurs sources: scripturae, cedulae pour les authentiques, sculpturae pour des inscriptions. Ils partagent presque à chaque fois leurs déceptions de paléographes, les authentiques illisibles ou qu'au contraire on ne peut plus faire correspondre aux reliques dispersées dans les reliquaires. Ils évoquent alors, selon les cas, "beaucoup d'autres reliques de nombreux saints", dont "Dieu sait les noms", dont les noms "sont inscrits dans le livre de vie" ${ }^{42}$, "dans le ciel" trouvé. Dire ces échecs permettait par contraste d'affirmer que tous les noms avancés repo-

\footnotetext{
${ }^{38}$ DUBOIS, Le trésor des reliques, reliquaire $\mathrm{n}^{\circ} 2$ : in uno parvo vasculo quandrangulari, inveni scriptum...

${ }^{39}$ On pourrait encore confronter un inventaire de 133 reliques rédigé en 1192 à la cathédrale de Sens (Cf. Prou, Chartraire, Authentiques de reliques, p. 131 et éd. p. 135-140) aux 137 authentiques lisibles antérieures au XII siècle (éd. ibid.), auxquelles s'en ajoutent encore d'autres publiées depuis in Chartae latinae, t. 19, $\mathrm{n}^{\circ} 682$.

${ }^{40}$ DuBOIS, Le trésor des reliques, $\mathrm{n}^{\circ} 5,13,14,25$.

${ }^{41}$ Ibid., $\mathrm{n}^{\circ}$ 6, 8 .

${ }^{42}$ Cf. entre autres Ap 20,12.15. Sur cette image, Leo KOEP, Buch IV (himmlisch), in Reallexikon für Antike und Christentum, Stuttgart, Hiersemann, 2, 1954, col. 725-732.

${ }^{43} \mathrm{Cf}$. entre autres Lc 10,20. Autre exemple dans un inventaire de l'abbaye bénédictine de Tholey au début du XIII siècle: preterea multorum sanctorum reliquie quorum nomina scripta sunt in conspectu domini. Ed. in Mittelalterliche Schatzperzeichnisse, t. 1, Von der Zeit Karls des Großen bis zur Mitte des 13. Jahrhunderts, éd. par le Zentralinstitut für Kunstgeschichte en collaboration avec Bernhard BISCHOFF, Munich, Prestel-Verlag, 1967, n 90.
} 
saient sur la lecture scrupuleuse des documents. Et loin de faire perdre leur valeur aux reliques restées anonymes, ces formules vagues laissent supposer d'autres richesses: un inventaire rédigé au milieu du XII ${ }^{\mathrm{e}}$ siècle à l'abbaye bénédictine de Muri affirme qu'il faut justement conserver les reliques sans authentiques avec d'autant plus d'attention qu'on ignore de qui elles sont ${ }^{44}$.

\section{Commémorer, légitimer, réformer par l'inventaire}

Les récolements généraux de reliques, opérations longues et parfois ardues, ont été entrepris dans plusieurs types de situation. Certains inventaires compilent des documents récents, suite à d'importantes acquisitions dont on souhaitait fixer la mémoire par écrit. C'est ce que fit entre 800 et 814 l'abbé Angilbert de Centula (aujourd'hui Saint-Riquier, Somme). Il rédigea lui-même un des premiers grands inventaires avec quelques 173 reliques, inclus dans un ouvrage où il faisait le bilan de ses activités de rénovation ${ }^{45}$. Un inventaire de 242 reliques de l'abbaye de Reading en Angleterre, inscrit sur quatre pages d'un cartulaire des années 1190, soit seulement soixante-dix ans environ après la fondation, peut aussi être considéré comme un premier regard en arrière après une période de forte croissance $^{46}$. On verra plus loin que de nombreuses copies de notices de consécration d'autels s'inscrivent dans cet esprit.

La rédaction d'un inventaire pouvait aussi permettre de sécuriser un ensemble de reliques jugées en danger, ou pour le moins de montrer qu'on les administrait avec rigueur. Un dossier passionnant à cet égard est celui des deux inventaires rédigés concurremment, à l'abbaye bénédictine de Zwiefalten, par le bibliothécaire Ortlieb et par le trésorier Berthold $^{47}$. Citations bibliques à l'appui, Ortlieb dénonce, dans la chronique du monastère qu'il

44... reliquie aliorum quorundam sanctorum, cum quibus breves non inveniantur, quas eo magis oportet caute servare, quo nescitur, cuius sanctitatis cuiusve meriti sunt. Ed. ibid., $\mathrm{n}^{\circ} 57$.

45 Angilbert de SaInT-RIQuier, De ecclesia Centulensi libellus, éd. G. Waitz, MGH SS 15,1, Hanovre, Hahn, 1887, p. 173-179. Waitz a édité ce texte aussi appelé De perfectione et dedicatione Centulensis ecclesiae d'après un manuscrit de la fin du XI $\mathrm{X}^{\mathrm{e}}$ siècle (Bibliothèque du Vatican, fonds de la reine Christine, ms. 235). Il fut aussi inséré, avec des variantes mineures, dans le Chronicon Centulense composé par Hariulf à la fin du XI ${ }^{e}$ siècle également: 1. II, c. 8-10 (catalogue des reliques au c. 9), éd. Ferdinand LOT, Hariulf. Chronique de l'Abbaye de Saint-Riquier, Paris, Picard, 1894, p. 61-67.

${ }^{46}$ British Library, ms Egerton 3031, f. 6v-8r. Cf. Denis BETHELL, The making of a Twelfth-Century Relic Collection, in G. J. CumIng et Derek BAKER (éd.), Popular Belief and Practice: Papers read at the Ninth Summer Meeting and the Tenth Winter Meeting of the Ecclesiastical History Society, Cambridge, Cambridge University Press, 1972, p. 64-72 (sans éd.). L'auteur tente de reconstituer à partir des noms quelles collections antérieures ont pu être mises à profit pour réunir ces reliques aussi rapidement.

${ }^{47}$ Cf. à ce sujet la bonne étude de Herrad SPILLING, Sanctarum reliquiarum pignera gloriosa. Quellen zur Geschichte des Reliquienschatzes der Benediktinerabtei Zwiefalten, Bad Buchau, Federsee-Verlag, 1992, p. 1-8. Sur une acqui- 
est en train d'écrire vers 1135-1137, la mauvaise gestion des reliques par Berthold. Il établit ensuite la liste des reliques dans les reliquaires et les monstrances, sans doute d'après des listes existantes et de mémoire. Mais son inventaire s'interrompt abruptement avant la liste annoncée des reliques sans monture. Ortlieb la destinait à être modifiée en soulignant de rouge les reliques qu'on aurait pu mettre à l'abri, en précisant la nature du nouveau reliquaire. Tout porte à croire que le trésorier Berthold ne permit pas à Ortlieb, qui l'attaquait directement, d'étudier de près ces reliques. Bien plus, Berthold entreprit à son tour en 1137 la rédaction d'une chronique, où il inséra un inventaire des objets dont il avait la garde, insistant sur ses acquisitions, recourant lui aussi aux citations bibliques, mais évitant tout détail sur les reliques qui faisaient l'enjeu du conflit.

Un inventaire des reliques de l'abbaye cistercienne de Kamp (Bas-Rhin) fut réalisé en avril 1472 sous l'abbé réformateur Henri de Ray $(1452-1483)^{48}$. Reliques et ornements avaient dû être évacués du monastère pour presque dix-huit mois en 1469-1470, dans la crainte d'un siège devant se tenir à dix kilomètres de là ${ }^{49}$. Réagissant sans doute à cette situation extrême, l'abbé fit aussi réorganiser les archives, si bien que la rédaction de l'inventaire s'inscrit clairement dans une entreprise plus vaste de sécurisation et de réforme. Un scandale impliquant une série de monastères cisterciens du Bas-Rhin dans une entreprise de commerce de reliques éclata un peu plus $\operatorname{tard}^{50}$. Il semble qu'il ait épargné Kamp, où l'on avait justement fait cet inventaire, mais l'affaire détermina sans doute l'abbé successeur, Heinrich von der Heyden (1483-1499), à associer à l'inventaire cinq extraits de textes normatifs compilés sous le titre Inbibitio de reliquiis sanctorum non distrabendis ${ }^{51}$. Ce sont trois instructions relatives à l'aliénation et aux conditions de conservation des reliques, données entre 1465 et 1483 par de hauts dignitaires cisterciens à l'occasion de visitations de Kamp; un extrait des diffinitionibus ordinis Cisterciensis indiquant qu'il faut poser les reliques sur les autels, avec des lampes ou des cierges, lors des principales fêtes et de celles de chaque saint; et un passage du Rational des divins offices où Durand de Mende explique les trois raisons d'exposer le trésor de l'église les jours de grande solennité: montrer la précaution qu'on a à le garder, la solennité elle-même, et la mémoire des donateurs. L'adjonction à l'inventaire

sition un peu ultérieure: Edina BOZOKY, Le trésor de reliques de l'abbaye de Zwiefalten. Un précieux témoignage des échanges culturels entre Orient et Occident, in Les échanges culturels au Moyen Age: formes et enjeux, Actes du XXXII ${ }^{\mathrm{e}}$ congrès de la Société des Historiens Médiévistes de l'Enseignement Supérieur Public, Boulogne-sur-Mer, 18-20 mai 2001, Paris, Publications de la Sorbonne, 2002, p. 115-133.

${ }^{48}$ Ed. Hans MOSLER, Das Camper Reliquienverzeichnis von 1472, in Annalen des Historischen Vereins für den Niederrhein, insbesondere das alte Erabistum Köln, 168/169, 1967, p. 60-101 (le texte p. 85-101).

49 Ibid., p. 81 note 73, qui renvoie à la chronique de Kamp (1483), où l'inventorisation n'est pas mentionnée.

${ }^{50}$ Ibid., p. 60 et $80-81$.

${ }^{51} \mathrm{~A}$ ce que je comprends, l'abbé Heinrich a relié ce document administratif à la suite de l'inventaire original, avec la chronique rédigée par lui en 1483 . Heinrich a par ailleurs compilé une liste en langue vulgaire et un calendrier des indulgences, cf. ibid., p. 77 n. 55; éd. de la liste p. 101. Le recueil ainsi constitué se trouve aux Archives de l'église paroissiale de Kamp. 
de ces textes de mise en garde, adressés à ses utilisateurs, rehausse encore son rôle d'outil de sécurisation de la collection.

Outre la commémoration et la sécurisation, la rédaction d'un inventaire pouvait permettre de remettre au jour des richesses oubliées, ce qui pouvait donner l'occasion d'un renouveau du culte. Il ne semble pas que cette démarche ait toujours été de soi. C'est "à la demande des fidèles", et le jour de la fête du saint, que l'abbé Erlebald ouvrit la châsse de Simètre dont il était question plus haut, sans que l'on sache bien ce qui motiva la demande $^{52}$. Sans doute est-ce qu'il fallait des circonstances particulières pour qu'on ose entrebâiller des reliquaires que l'on avait toujours vus fermés. Si certaines reliques étaient bien connues et même visibles dans leurs écrins, d'autres déballages ont pu provoquer des réactions qui, dans les descriptions, s'apparentent à de véritables terreurs sacrées ${ }^{53}$ : le contenu de certains reliquaires était réputé aussi puissant qu'il était mystérieux. Ceci explique l'impression que les récolements sont finalement relativement rares, tout au moins avant la fin du Moyen Age, où l'activité administrative et gestionnaire aura souvent surmonté l'éventuelle crainte du sublime.

A l'abbaye du Mont-Saint-Michel en Normandie, l'abbatiat de Pierre le Roy (13861410) fut une époque de réorganisation administrative et liturgique après la première phase de la guerre de cent ans. Après une visitatio, un moine anonyme rédigea en 1396 un inventaire des reliques ${ }^{54}$ : environ 200 , dans une quarantaine de reliquaires ${ }^{55}$. Certains abritaient une relique unique, tandis que d'autres contenaient d'importantes séries, ainsi plus de 45 dans un seul coffret. Il s'agit alors de la part la moins vivante de la collection, des reliques acquises anciennement puis délaissées dans un certain désordre. De tels inventaires avaient pour effet d'en rappeler l'existence, étape préalable à leur remise en valeur. Dans la marge de l'inventaire de Brunswick de 1482, qui recense pas moins de 1220 reliques, figure plusieurs fois le mot nota, indiquant très probablement l'intention de réaliser de nouveaux reliquaires pour des reliques jugées importantes ${ }^{56}$.

\footnotetext{
${ }^{52}$ L'inventaire se termine par: Idem abbas petitione fidelium ecclesie, Harduini videlicet inuestiti ecclesie de Lernau sarcofagum in quo reliquie he continebantur; idem abbas apperuit et has predictas reliquias invenit.

${ }^{53}$ Cf. à Brunswick les scrupules exprimés par l'abbé Bertold de Saint-Aegidius dans son récit d'une ouverture de châsse en 1460: BoOCKMann, Die verlorenen Teile, p. 57.

${ }^{54}$ Aujourd'hui connu par la copie qui en fut faite, au XVe siècle, dans un manuscrit regroupant plusieurs textes relatifs à l'histoire du Mont: ms Avranches BM 213. Le titre complet est Ista que secuntur per modum inventorii inventa sunt de sanctis reliquiis in thesauraria Montis diligenti, bumili, devota et reverenti visitatione facta a pluribus devotis et fide dignis anno Domini $\mathrm{M}^{\circ} \mathrm{CCC} \mathrm{C}^{\mathrm{mo}}$ nonagesimo sexto. Je m'appuie sur DUBOIS, Le trésor des reliques, ici p. 503.

${ }^{55} \mathrm{~F}$. Neveux a compté 216 reliques en ajoutant à l'inventaire celles qui sont citées dans quelques documents antérieurs. Les inventaires du XVII siècle en recensent 246. Quant aux reliquaires, l'éditeur Dubois en a regroupé certains et obtenu 29 numéros. Le $\mathrm{n}^{\circ} 5$ mentionnant aliis parvis jocalibus, on ignore leur nombre exact. L'étude de F. Neveux s'appuie essentiellement sur celle de dom Dubois: NEVEUX, Les reliques, ici p. 249.

${ }^{56}$ BOOCKMAnn, Die verlorenen Teile, p. 60 et 70.
} 
Si la rédaction d'un inventaire permet l'actualisation et le renouvellement, la fabrication de nouveaux reliquaires s'inscrit bien évidemment dans le même esprit. A partir du Moyen Age central, certaines formes de reliquaires répondent au besoin d'accueillir des séries de reliques importantes de manière adaptée, sans les mélanger. Pour éviter que les particules ne se perdent, on leur ménageait des cases individuelles, où des authentiques bien visibles les désignaient sous leurs fenêtres de $\mathrm{crista}^{57}$, ou bien on les collait dans de la résine ou de la cire, technique attestée par l'inventaire de Kamp en $1472^{58}$ et par des restes matériels dans des reliquaires de Brunswick ${ }^{59}$. Sans doute est-ce que la multiplication de reliquaires monstrances de relativement faible valeur, dans les trésors d'églises de la fin du Moyen Age, s'explique autant par la nécessité de gérer décemment et de mettre en valeur des collections de reliques toujours croissantes, que par la volonté de les donner à "voir"

\section{Les structures des inventaires: raconter la collection}

Un inventaire n'est pas toujours une simple liste. Il peut être ordonné selon différents critères, qui fondent autant de modes de présentation d'une collection de reliques.

\section{Hiérarchie des saints et revendication d'universalité}

La répartition spatiale des reliques dans les autels est un premier élément structurant des inventaires de reliques. Le nombre des autels dans les grandes églises augmenta considérablement à partir du VII ${ }^{\mathrm{e}}$ siècle, dans tout l'Occident, à la suite d'un processus de concentration en un seul édifice des différents bâtiments qui jusqu'alors avaient constitués les groupes ecclésiaux. Jusqu'au $\mathrm{XI}^{\mathrm{c}}$ siècle, ces autels furent souvent disposés de manière à constituer par leurs titulatures une véritable géographie représentative de la hiérarchie

\footnotetext{
${ }^{57}$ Ainsi un reliquaire du $15^{\mathrm{e}}$ siècle, provenant de l'abbaye du Ronceray à Angers, aujourd'hui au trésor de la cathédrale. En forme de plaque quadrangulaire, il comporte 24 cases où sont restées 17 authentiques: deux des $12^{\mathrm{e}}$ et $13^{\mathrm{e}}$ siècles, 15 contemporaines de sa réalisation. Apparemment de la même main, celles-ci sont écrites à l'encre rouge pour certaines, bleue ou dorée pour la plupart, avec un jeu dans les couleurs de lettres: manifestement destinées à être vues, elles faisaient partie du décor de l'objet. Leur disposition fixe fait de l'ensemble une sorte d'inventaire. Certaines indiquent d'ailleurs des reliques anonymes, les autres de un à trois saints. Cf. Ch. URSEAU, Authentiques de reliques provenant de l'ancienne abbaye du Ronceray à Angers, in Bulletin bistorique et philologique du Comité des travaux bistoriques et scientifiques, 1903, p. 587-593; pour le reliquaire: Monique JACOB, et al., Les orfêves d'Anjou et du bas Maine, Paris, Editions du patrimoine, 1998 (Cahiers du patrimoine, 50), p. 326, ill.

${ }^{58}$ Reliquie... cum cera alba ibidem imposite. MOSLER, Das Camper Reliquienverzeichnis, $\mathrm{n}^{\circ} 33$.

${ }^{59}$ Cf. Boockmann, Die verlorenen Teile, p. 65.

${ }^{60}$ Cf. Christof L DiEDriCHS, Vom Glauben zum Sehen. Die Sichtbarkeit der Reliquie im Reliquiar. Ein Beitrag zur Geschichte des Sehens, Berlin, Weißensee Verlag, 2001.
} 
céleste $^{61}$. Or l'habitude s'était établie de déposer des reliques dans tous les autels, même annexes. Les reliques étant choisies au moins en partie en fonction de la titulature des autels, l'ordonnancement spatial des autels impliquait les reliques dans un système narratif $f^{62}$. Avant la consécration de nouveaux autels, il fallait recenser les reliques disponibles pour les classer et les partager entre les autels où elles seraient déposées. Rédigés dans ce cadre, les inventaires de reliques inclus dans les notices de consécration donnent donc une image d'un ensemble fraîchement réorganisé, à la différence des récolements généraux qui peuvent jeter une lumière soudaine sur plusieurs siècles de désordre accumulé.

Les répartitions de reliques dans les autels sont parfois difficiles à interpréter aujourd'hui, d'autant plus qu'au-delà d'un certain seuil quantitatif, une part d'arbitraire semble évidente: tous les dépôts ne pouvaient plus faire sens. Ce seuil physique étant atteint, il restait toujours possible de rendre la collection significative indépendamment de son déploiement dans l'espace, au moyen d'un texte d'inventaire. Dans l'état de notre documentation, le premier ou un des premiers à le faire parait être, au début du $\mathrm{IX}^{\mathrm{e}}$ siècle, Angilbert de Saint-Riquier. Il classe ses 173 reliques selon la hiérarchie universelle des saints, c'est-à-dire qu'après celles du Christ et de la Vierge, on trouve d'abord les apôtres, puis les martyrs, les confesseurs (qui ne connurent pas le martyre), et les vierges ${ }^{63}$. Cette hiérarchie est une typologie historique grossière, puisqu'elle décrit les différents modes de vie et de mort qui déterminèrent l'accès à la sainteté avant et après l'officialisation du christianisme. Elle permet surtout de mettre en avant l'universalité d'une collection, en montrant que chaque type de saint y est représenté. Utilisée de manière extrêmement fréquente, c'est la première qui vient à l'esprit des auteurs d'inventaire de reliques.

Il me semble très probable que la présentation hiérarchique des reliques dans les inventaires ait été empruntée aux litanies. Née sans doute en Orient au IV ${ }^{e}$ siècle, cette forme de prière d'intercession s'était établie au VII siècle chez les Anglo-saxons et les Irlandais. Diffusée ensuite par les missionnaires, elle fut adoptée dans le monde franc vers le milieu du VIII ${ }^{\mathrm{e}}$ siècle. Or il semble justement qu'Angilbert, ainsi que son maître Alcuin à l'école du palais d'Aix-la-Chapelle, aient pris part activement à cette diffusion. Vers 800 , Alcuin fut peut-être à l'origine de l'adjonction d'une litanie dans un psautier du monastère féminin de Notre-Dame de Soissons. Avec un peu moins de 200 noms, cette liste est de loin la plus longue des quatre litanies franques conservées antérieures à $800^{64}$. Angilbert luimême préconise, dans son Institutio de diversitate officiorum rédigé alors qu'il était abbé de

\footnotetext{
${ }^{61}$ Günter BANDMANN, Früh- und hochmittelalterliche Altaranordnung als Darstellung, in Victor H. ELBERN, (éd.), Das erste Jahrtausend. Kultur und Kunst im werdenden Abendland an Rhein und Rubr, t. 1, Düsseldorf, Schwann, 1964 , p. 371-411.

${ }^{62}$ Ceci peut d'ailleurs aller au-delà des seuls autels puisque des reliques étaient également placées au sommet des colonnes, des clochers, etc. Cf. HERRMANN-MASCARD, Les reliques, p. 169-173.

${ }^{63}$ ANGILBERT, De ecclesia Centulensi libellus.

${ }^{64}$ Michael LAPIDGE (éd.), Anglo-Saxon Litanies of the Saints, Londres, 1991 (Henry Bradshaw Society, 106), p. 35-36.
} 
Saint-Riquier, l'emploi liturgique de diverses litanies, et il possible qu'il en ait lui-même compilé.

Comme les litanies, les inventaires de reliques sont d'abord des listes de noms de saints, et on verra plus loin l'usage dévotionnel ou liturgique qu'on fit parfois d'eux. Une certaine perméabilité entre ces deux types de texte est donc assez naturelle. Guillaume Durand écrit explicitement à la fin du XIII ${ }^{\mathrm{e}}$ siècle que lors des consécrations d'autel, on chantait des litanies de saints incluant ceux dont on déposerait les reliques ${ }^{65}$. Mais il y avait surtout un troisième type de texte, qui se développa durant la seconde moitié du VIII ${ }^{e}$ siècle dans le monde franc à partir des litanies: ce sont les Laudes Regiae, acclamations au souverain vers lequel on oriente les bienfaits des saints énumérés par séries ${ }^{66}$. Or Angilbert de Saint-Riquier était justement un proche du souverain. Gendre de Charlemagne, il affirme avoir reçu une partie de ses reliques de la chapelle impériale. Plus encore, le récit qu'il fait, toujours dans le même texte, du reste de ses acquisitions, semble avoir pour but d'exposer les bons rapports qu'il entretenait lui-même avec tous les puissants imaginables, autant de donateurs. Tout comme le détournement des litanies au profit du roi dans les Laudes Regiae, le rassemblement de reliques en collections d'une ampleur qui paraît inouie jusque là, et leur présentation universaliste dans de tels inventaires ${ }^{67}$, sont des éléments de la politique carolingienne du pouvoir, largement liée au prestige ecclésiastique.

\section{Les objets et l'bistoire, l'écrit et l'oral}

Les reliques sont objets d'histoires, celles des personnes dont elles sont les restes, et celles de leur parcours et de leur arrivée en un lieu donné. On a appelé cela plus haut leurs biographies, que les historiens d'aujourd'hui tentent de reconstituer. On trouve déjà cette démarche dans certains inventaires de reliques, qui se présentent alors comme des textes historiographiques, comme des scenarii à dérouler pour dire et éprouver l'histoire à travers une mise en scène d'objets.

Un inventaire à la structure assez particulière, quelque peu impressionnant sur ce plan, fut rédigé parallèlement à une chronique par un moine bénédictin de Saint-Pierre-le-

\footnotetext{
${ }^{65}$ Et scola iterum incipit letaniam in qua bis nominetur sanctus ille in cujus nomine ecclesiae vel altare dedicatur et eorum quorum ibi reliquiae recluduntur. Le pontifical de Guillaume Durand, livre II ordo II c. 10, éd. ANDRIEU in Le pontifical romain, t. 3 p. 463 .

${ }^{66}$ Cf. à ce sujet Gisbert KNOpP, Sanctorum nomina seriatim. Die Anfänge der Allerheiligenlitanei und ihre Verbindung mit den "Laudes regiae", in Römische Quartalschrift für christliche Altertumskunde und Kirchengeschichte, 65,1970 , p. $185-231$, en particulier p. 212 et 224.

${ }^{67} \mathrm{La}$ collection de reliques de la chapelle du palais à Aix-la-Chapelle, commencée par Pépin et notablement amplifiée par son fils Charlemagne, n'est qu'imparfaitement connue par des inventaires fragmentaires, ce qui laisse paraître d'autant plus exceptionnel celui d'Angilbert. Cf. Heinrich SCHIFFERS, Karls des Großen Reliquienschatz und die Anfänge der Aachenfahrt, Aix-la-Chapelle, Volk, 1951.
} 
Vif de Sens, Geoffroy de Courlon. Achevée en 1295, la chronique ${ }^{68}$ était sans doute déjà avancée quand le prieur demanda à Geoffroy - qui au moins l'écrit ainsi - de préparer un manuel à l'usage du sacristain, pour aider celui-ci à répondre aux questions des visiteurs sur les reliques du monastère ${ }^{69}$. Il semble bien que la copie conservée, datée de 1293, ait justement été celle du sacriste ${ }^{70}$. Geoffroy emploie dans ce "livret sur les reliques" des informations réunies pour sa chronique, et le présente d'emblée comme un ouvrage historique. Après un inventaire assez classique, plus de 80 chapitres inscrivent chacune des reliques dans une histoire qui est finalement universelle et locale à la fois. Geoffroy commence par les mystères de la Trinité et de l'Incarnation, puis va d'une relique de la Passion à l'autre. Chaque fois, l'épisode relatif à l'objet est relaté, avant de préciser éventuellement qui apporta la relique au monastère et à quelle date, quand et comment elle y est présentée, et le cas échéant qui fit faire le reliquaire. Suivent la nativité et l'assomption de la Vierge, ses vêtements, ses cheveux, son lait. Après le récit de miracles survenus à l'abbaye, on passe aux saints, dans l'ordre hiérarchique, dont Geoffroy rappelle les actes. On a reconstitué qu'il avait puisé dans la Bible, les écrits de Jérôme, d'Augustin et Jean Chrysostome, la légende dorée, les évangiles apocryphes de Jean et Nicodème. Parmi d'autres documents, Geoffroy livre enfin des listes d'abbés, d'archevêques et de rois qui furent complétées jusqu'au XVIII ${ }^{\mathrm{e}}$ siècle, c'est-à-dire que le manuscrit fut utilisé cinq siècles. Ce texte est pleinement au service des objets, sans lesquels il n'aurait pas raison d'être. A travers eux, on veut transmettre aux visiteurs du monastère des récits d'histoire sainte et locale. Sans doute est-ce que le sacriste pouvait puiser dans l'ouvrage comme un prédicateur dans une collection de sermons. Mais cette communication n'a pas lieu dans les cadres habituels de la liturgie ou de la prédication, et va bien au-delà du simple pèlerinage

${ }^{68}$ GeOfFroy de COURLon, Chronique de l'abbaye de Saint-Pierre-le-Vif de Sens, éd. et trad. par Gustave Julliot, Sens, Duchemin, 1876 (Publications de la société archéologique de Sens, 2).

69 Dans le prologue à l'ouvrage: Inde est quod ego inmittis belua sagumque cilicinum videns predecessorum scripturas super reliquiis sanctorum et sanctarum in nostro monasterio Sancti Petri Vivi Senonensis honorifice servatis longevis temporibus jam transactis editas, adeo veteratas ut absque difficultate non valeant recognosci, quia sic viro religioso priori nostro placuit renovare etiam de prolixis nostri cenobii libris brevissime eligendo conscribere dignum duxi, ita tamen ut libellus a me minori inferiorique editus jussu predicti prioris penes sacristam remaneat, ut possit dictus sacrista postulantibus demonstrare que et quante sunt sanctorum sanctarumque reliquie que debent a fidelibus Christi in predicto Sancti Petri monasterio venerari. Ed. Gustave JULLIOT, Maurice Prou, Le livre des reliques de l'abbaye Saint-Pierre-le-Vif de Sens publié avec plusieurs appendices, Sens, Duchemin, 1887 , p. $2-3$.

${ }^{70}$ Paris BnF Nlle acq. lat. 311, éd. ibid. (sans les neumes de l'office). C'est un livre de parchemin de taille maniable $-20,6 \mathrm{~cm}$ de haut sur 14 de large, 72 folios -, contenant après le libellus des reliques jusqu'au f. $48 \mathrm{r}$, un office de sainte Vénère, et un calendrier destiné à servir d'Ordo missarum. On ne s'explique pas bien la présence de cet office chanté le 26 juillet, mais les autres textes s'adressent clairement au sacriste. Le libellus et le calendrier furent copiés par une main qui n'est pas celle de l'auteur, mais celui-ci a pu apporter des corrections au calendrier et à la liste des archevêques de Sens incluse dans le libellus, cf. ibid. p. XV. Une seconde copie du libellus, datée 1294, était reliée devant un exemplaire de la chronique, dans un manuscrit perdu signalé en 1660. Cf. Chronique, p. VI-IX et Le livre des reliques, p. 1. 
par le nombre des objets concernés et la richesse des récits. Qui étaient ces visiteurs, où les reliques leur étaient-elles montrées ? Le livre de Geoffroy ne nous le dit pas.

La dimension historique de l'inventaire de reliques réalisé en 1396 au Mont-SaintMichel est plus subtile, ce critère n'étant pas le seul qui présida à sa rédaction. Le texte commence par le don le plus ancien, un autel portatif passant (dicitur) pour un don du Saint-Siège lors de la fondation de l'église ${ }^{71}$, et se clôt sur le plus récent, un don de reliques dont celle de la croix par Charles VI en 1395. Voilà qui fixe un cadre, entre le pape et le roi de France. La hiérarchie des saints n'est plus déterminante, l'histoire du reliquaire ou de la relique prenant le pas sur celle de la personne qu'elle représente.

Pour commenter les objets, l'auteur s'appuie sur des sources diverses, écrites autant qu'orales. Essayons de les repérer. Il renvoie à une charte scellée par l'évêque de Tréguier et à des documents délivrés par les frères mineurs pour légitimer l'origine d'un groupe de reliques provenant de Guingamp, offertes par le comte Henri de Bretagne en $1388^{72}$. L'article suivant cite un reliquaire donné par le père du comte. Celui-ci n'est pas nommé, alors que ce reliquaire portait l'inscription C'est la coste de s. Yves que Monsieur Charles de Bloys y donna, selon un inventaire de 1647. L'objet était important, Charles ayant financé la canonisation d'Yves en 1347. Mais jusqu'à sa mort en 1364, il avait tenté d'enlever le duché de Bretagne, auquel son fils Henri renoncerait en 1365: l'oubli de 1396 pourrait, selon J. Dubois, être un égard au duc régnant. D'autres objets appellent d'autres commentaires. Pour les reliques du fondateur, saint Aubert, on fait référence à sa légende ${ }^{73}$. Aucun renvoi à un texte écrit, par contre, pour un fragment du voile apporté du Paradis et déposé sur un autel, en signe de dédicace, par l'archange saint Michel. Mais au sujet de l'histoire de l'écu et l'épée avec lesquels il tua un dragon, l'auteur se réfère à la chronique que l'archevêque Baudry de Dol écrivit au XI ${ }^{\mathrm{e}}$ siècle ${ }^{74}$. C'était alors le prieur du Mont, qui la tenait lui-même de vieux moines connus enfant, qui la lui avait racontée. En 1396, l'écrit supportait depuis longtemps le récit oral.

${ }^{71}$ Cf. DuboIs, Le trésor des reliques, $\mathrm{n}^{\circ}$ 1: Et dicitur istud altare a Sede Apostolica in prima doctatione istius ecclesie visis tot et tantis signis et miraculis, devote fuisse transmissum. La nature des reliques contenues dans l'autel indique une provenance romaine du IXe siècle au plus tôt.

${ }^{72}$ Ibid., $\mathrm{n}^{\circ} 10:$... habemus instrumentum publicum confirmatum sigilli episcopi Tricor(ensis) et literas patentes a fratre gardiano Fratrum Minorum.

${ }^{73}$ Ibid., $\mathrm{n}^{\circ} 16: \ldots$ nam pro legitur in antiquis istius sancti loci miraculis...; $\mathrm{n}^{\circ} 17: \ldots$ ut diffuse patet in antiquis istius sancti loci scripturis et bystoriis, et maxime in legenda ipsius beati confessoris Auberti, de qua diffusius patet in sequentibus quomodo inventa sunt.

${ }^{74}$ Ibid., $\mathrm{n}^{\circ}$ 27-28: Item portio notabilis pallioli in descensu beati archangeli a paradiso allati, et supra unum altare in ecclesia in signum dedicationis ipsius ecclesiae ibidem relicti. Item, babemus preciosum et intersignum et pignus, scutum cum gladio vel ense supra drachonem precepto divino a beato Michaele interfectum, et ad bunc sanctum locum miraculose delatum, ut patet ex narratione domini Baldrici, Dolensis archiepiscopi, intus reservata. 
Mises à part les authentiques de reliques et quelques listes préexistantes, les sources de l'inventaire réalisé entre 1489 et 1499 à l'abbaye de Zwiefalten semblent être au contraire exclusivement orales ${ }^{75}$. L'auteur, qui écrit soigneusement mais s'exprime dans un latin approximatif, a omis de dater son inventaire. Ce récolement est un pur outil administratif, ignorant des inventaires inclus au XII ${ }^{\mathrm{e}}$ siècle dans les chroniques d'Ortlieb et Berthold, évoqués plus haut. Cette méconnaissance des sources extérieures à la collection elle-même, ou plutôt cette absence de recherche supplémentaire, confère un relief particulier aux rares traces de savoir écrit qu'on peut repérer dans ce texte non historiographique. Il devait s'agir alors d'un savoir élémentaire, partagé par tous les membres de la communauté. L'inventaire s'ouvre sur la notice de consécration de l'église, dont le texte exact n'est pas conservé, le plus proche témoin en étant aujourd'hui la chronique de Berthold. L'auteur de l'inventaire écrit semptenbris pour septembris, ce qui laisse penser qu'il a transcrit ce texte de mémoire plutôt qu'il ne l'aurait copié. Il introduit le passage par anno domini, une formule facilitant la mémorisation qui revient constamment dans les Annales du monastère. Une référence explicite à cet ouvrage est faite au sujet de l'origine de reliques des 11000 vierges: là encore, le texte ne correspond pas exactement et ressemble fort à la transcription maladroite d'un savoir oralisé. Or les Annales étaient reliées avec le martyrologe de l'abbaye, ce qui pourrait bien indiquer que le manuscrit se trouvait dans la salle capitulaire, et qu'on en lisait des extraits devant tout le monde à l'office du chapitre ${ }^{76}$. Ayant reconnu le rôle de l'oralité dans la rédaction de cet inventaire, on peut déceler quels savoirs historiques étaient alors les plus vivants, et notamment apprécier l'efficacité des stratégies mémorielles concurrentes mises en œuvre, plus de trois cent ans auparavant, par les chroniqueurs Ortlieb et Berthold. Après s'être réglé des comptes par inventaires interposés comme on l'a vu, ils avaient organisé une translation de reliques chacun, et rédigé un texte la relatant. $\mathrm{Si}$ la critique historique était appelée à témoigner, elle tenterait aujourd'hui de défendre Ortlieb le bibliothécaire. Mais c'est d'abord Berthold le trésorier qui, malgré sa gestion apparemment douteuse, fut vainqueur devant l'Histoire, puisque c'est de "sa" translation de reliques que le rédacteur de l'inventaire s'est souvenu à la fin du $\mathrm{XV}^{\mathrm{e}}$ siècle, et non de celle opérée par Ortlieb.

Citons encore un inventaire rédigé entre le début du XII ${ }^{\mathrm{e}}$ et le milieu du XIII ${ }^{\mathrm{e}}$ siècle, qui a ceci de particulier de ne pas décrire les reliques d'un lieu donné: intitulé Index insigniorum reliquiarum, le texte énumère les plus importantes reliques de la Chrétienté, éla-

\footnotetext{
${ }^{75} \mathrm{Je}$ m'appuie ici sur SPILLING, Sanctarum reliquiarum .

${ }^{76}$ Sur l'office du chapitre et ses livres, voir Jean-Loup LEMAITRE, Mourir à Saint-Martial. La commémoration des morts et les obituaires à Saint-Martial de Limoges du XI au XIII siècles, Paris, De Boccard, 1989.
} 
borant un récit géographique plutôt qu'historique. Une insistance sur l'Aquitaine laisse cependant soupçonner une origine poitevine ${ }^{77}$.

\section{Le système descriptif des reliquaires}

Le classement des reliques dans l'ordre hiérarchique des saints est généralement incompatible avec une description reliquaire par reliquaire. Il existe certes une formule intermédiaire, appliquée à Kamp en $1472^{78}$ : après avoir dépouillé les authentiques, on a pris la peine de classer les noms des reliques enfermées dans chacun des 34 reliquaires, soit quelques 800 particules relatives à 292 personnes. Mais même comme cela, la structure du texte reste déterminée par l'ordre des reliquaires. Celui-ci peut être apparemment anarchique ou répondre à plusieurs critères, parfois simultanément. L'importance attribuée à certains objets, leur signification historique si l'on veut, en est un comme on l'a vu au MontSaint-Michel.

De nombreux inventaires classent plus simplement les reliquaires selon leurs formes. A Saint-Blaise de Brunswick en 1482 par exemple, on a traité par groupes, après les six principaux objets, les 26 coffrets, les 22 monstrances et 28 autres reliquaires-monstrances, les dix bras reliquaires, etc. Les monstrances apparaissent par ordre de taille, au centimètre près si l'on en juge d'après celles qui sont conservées, relativement nombreuses ${ }^{79}$. Ces typologies formelles correspondraient-elles à des systèmes de rangement dans les sacristies? Ce n'est pas exclu, puisque l'évolution générale des inventaires, dont il faudra bien sûr nuancer l'appréciation en les étudiant plus nombreux, semble aller de textes narratifs et littéraires vers des textes plus descriptifs et objectifs.

En tant qu'outils de gestion, les inventaires devaient permettre de distinguer les reliquaires les uns des autres. Cela n'empêche pas que les descriptions soient courtes, car elles s'adressaient à de très bons connaisseurs de ces objets, qui les fréquentaient au cours de longues années. L'inventaire de Kamp, de 1472, considère quatorze monstrances. Certaines sont qualifiées sommairement, comme argentea cum rotundo cristallo, ou cum tribus turribus, d'autres sont désignées par leur petite taille et dites monstranciola, etc. Mais le vocabulaire descriptif des auteurs n'a pas toujours suffi. Ils ont alors recouru à un système de signes apposés sur les objets. Une monstrance dite signata cum craticula est donc marquée d'une petite grille, deux autres sont signata cum tali signo, lesdits signes étant copiés dans la marge,

\footnotetext{
${ }^{77}$ BnF lat. 14069, manuscrit originaire de Saint-Germain-des-Près, f. 178-180. Cf. Pierre BATIFFOL, Inventaire de reliques provenant d'un $\mathrm{ms}$. du XIIII] ${ }^{\mathrm{e}}$ siècle, in Bulletin de la Société nationale des antiquaires de France, 1891 , p. $218-225$, éd. p. 220-225.

${ }^{78}$ Cf. MOSLER, Das Camper Reliquienverzeichnis.

${ }^{79}$ Boockmann, Die verlorenen Teile, p. 52.
} 
par exemple une étoile à huit branches surmontée d'un calice ${ }^{80}$. Ce système devait être poussé plus loin, avec des images des objets eux-mêmes, lorsque les reliquaires étaient plus nombreux ou l'inventaire plus soigné: ainsi à Brunswick en 1482 et de manière plus systématique et spectaculaire à Bamberg en 1508-1509 et surtout à Halle à la fin des années $1520^{81}$.

On a souvent été déçu par les inventaires médiévaux, textes administratifs et historiques qui paraissent trop secs. Ils sont étrangers à la tradition antique et réactualisée à la Renaissance de l'ekphrasis, exercice littéraire de description des œuvres qui deviendra une condition de l'analyse en histoire de l'art. Mais ces inventaires sont, à leur manière, des descriptions d'experts qu'il faut prendre au sérieux. Ils sont un moyen unique d'approcher les objets avec des yeux médiévaux, en évitant certains anachronismes du regard, c'est-àdire en apprenant à résister aux fioritures orfévrées pour se laisser guider vers les reliques elles-mêmes, éprouver l'empathie qu'il faut, et raisonner à ce niveau.

\section{Supports et diffusion des inventaires: la médiation des collections}

Les différents supports sur lesquels les inventaires de reliques ont été transmis témoignent de leur fonction, et par là même de celle des collections d'un établissement donné, dont ils présentaient une image élaborée.

Il semble que beaucoup d'inventaires à usage administratif et interne, d'une circulation très réduite, étaient inscrits en un exemplaire unique sur une feuille ou un cahier. Sans doute est-ce que les documents périmés étaient facilement détruits ou perdus, et qu'il en reste peu par rapport à ce qui a pu exister. Trois feuilles de parchemin provenant d'Engelberg en Suisse pourraient témoigner d'un renouvellement des inventaires dû à une accumulation rapide de reliques. Sur la première ont été inscrits, d'une écriture du $\mathrm{XI}^{\mathrm{e}}$ ou du XII siècle, environ 90 noms; les deux suivantes, du XII siècle, citent un peu plus de 150 reliques puis un peu moins de $200^{82}$. Sur la couverture de l'inventaire réalisé en 1482 à Brunswick, une inscription indique qu'il était destiné à rester avec les reliques, dans l'autel majeur, qui devait donc comporter une armoire ou petite pièce servant de sacristie ${ }^{83}$.

\footnotetext{
${ }^{80}$ MOSLER, Das Camper Reliquienverzeichnis, $\mathrm{n}^{\circ} 8,10$ et 11 . Sur des objets conservés, il ne faudrait pas confondre ces signes avec des poinçons d'orfèvres ou de villes!

${ }^{81} \mathrm{Je}$ me permets de renvoyer à Philippe CORDEZ, Die Wallfahrt als Medienereignis. Korpus und Deutungsversuche der Heiltumsverzeichnisse mit Reliquienbildern im Heiligen Römischen Reich (1460-1520), in Andreas TACKE (dir.), "Ich armer sundiger Mensch". Heiligen- und Reliquienkult in der Zeitenwende Mitteldeutschlands, Göttingen, Wallenstein, à paraitre en 2006.

82 Ed. STÜCKELBERG, Geschichte der Reliquien, t. 1, p. XXXIX-XL.III.

${ }^{83}$ BOOCKMANN, Die verlorenen Teile, p. 43. Titre complet: Registrum in quo conscripte sunt Reliquie que habentur in / ecclesia sancti Blasii Brunswicensis Et manebit / in altari circa reliquias. Les comptes de l'armarium indiquent en 1476
} 


\section{Chroniques et inscriptions monumentales}

De nombreux inventaires ont été conservés parmi des documents réunis pour leur valeur historique, ce qui indique un changement de fonction ou une double fonction, entre administration et commémoration. Cela était particulièrement naturel pour les notices de consécration, enfermées dans des autels jusqu'à l'ouverture qui les rendait caduques. Pour qu'une notice de consécration valide soit aussi lisible, il fallait la transcrire sur un autre support. Un inventaire des reliques conservées dans cinq autels de l'abbaye de Pfävers, rédigé et inscrit vers 870 sur une page de garde ajoutée à un lectionnaire, est sans doute l'extrait d'une notice de consécration ${ }^{84}$. De telles reprises sont fréquentes, fondues dans des textes à caractère de chronique ou portées isolément dans des manuscrits divers ${ }^{85}$.

L'importance politique de certaines consécrations a aussi pu déterminer la reprise totale ou partielle des notices. En plus des évêques consécrateurs, parfois nombreux, de grands laïcs pouvaient participer à ces événements. Liée au dispositif des titulatures d'autels, la mise en scène des actes de chacun a pu assumer des significations stratégiques qu'il fallait fixer par écrit. Ce fut le cas des rituels de consécration des cathédrales de Halberstadt en 992 en présence d'une dizaine d'évêques, de princes, de l'empereur Otton III et de sa cour, de Bâle en 1019 en présence d'Henri II, de Saint-Servais de Quedlinburg en 1021. Leurs descriptions, copiées et recopiées dans des chroniques, soit aussitôt, soit deux ou quatre siècles plus tard, comprenaient aussi des listes de reliques ${ }^{86}$.

un paiement pour la réalisation d'un verrou "derrière l'autel majeur où sont les reliques" (retro summum altare ubi recludent reliquiae): p. 50 note 182. Cf. sur ce type de dispositif BRAUN, Der christliche Altar, t. 1, p. 212-220.

${ }^{84}$ Éd. Franz PERRET, Urkundenbuch der südlichen Teile des Kantons St. Gallen (Gaster, Sargans, Werdenberg), 1, Rorschach, Cavelti, 1951 , p. 50-52. Analyse (avec carte des provenances des reliques): Iso MÜLLER, Das Reliquien-verzeichnis, in Thesaurus Fabariensis. Die Reliquien-, Schat $\approx$ - und Bücherverzeichnisse im Liber Viventium von Pfäfers, Saint-Gall, 1986 (Sankt-Galler Kultur und Geschichte, 15), p. 13-55, ici en particulier p. 13 et Carl PFAFF, Die Schatzverzeichnisse, ibid., p. 57-84, ici p. 62, citant STÜCKELBERG, Geschichte der Reliquien, t. 1, n ${ }^{\circ}$ 46.

${ }^{85}$ Nombreuses listes de reliques reprises avec des notices de consécration parmi les quelques 80 Fundationes et dedicationes ecclesiarum éd. par Oswald HOLDER-EGGER et Georg WATTZ in MGH, SS, 15,2, Hanovre, 1888, p. $960-1125,1269-1285$ et 1315 ; d'autres parmi les 28 Dedicationes ecclesiarum germaniae et galliae éd. par Harry BRESSLAU in MGH, SS, t. 30/2, 1934, p. 768-788. Voir encore parmi les 162 notices de consécration sur supports divers rassemblées in Hermann TÜCHLE, Dedicationes Constantienses. Kirch- und Altarveihen im Bistum Konstanq bis zum Jahre 1250, Fribourg, Herder, 1949; ainsi que Wilhelm DEINHARDT (éd.), Dedicationes Bambergenses. Weibenotizen und-urkunden aus dem mittelalterlichen Bistum Bamberg. Mit einer geschichtlichen Einleitung, Fribourg, Herder, 1936 (Beiträge zur Kirchengeschichte Deutschlands, 1).

${ }^{86}$ Sur ces consécrations cf. Karl Josef BENZ, Untersuchungen zur politischen Bedeutung der Kirchweibe unter Teilnahme der deutschen Herrscher im boben Mittelalter. Ein Beitrag zum Studium des Verbältnisses zvischen weltlicher Macht und kirchlicher Wirklichkeit unter Otto III. und Heinrich II, Kallmünz, Michael Lassleben, 1975 (Regensburger historische Forschungen, 4), ici p. 176 (Quedlinburg), 234-236 (Halberstadt) et 159 (Bâle). 
Même un récolement général de reliques comme celui réalisé au Mont-Saint-Michel en 1396, qui il est vrai exprime par sa forme une vision historienne, a pu être copié au $\mathrm{XV}^{\mathrm{e}}$ siècle dans un manuscrit regroupant plusieurs textes relatifs à l'histoire du Mont ${ }^{87}$.

L'importance fondatrice accordée aux notices de consécration a pu leur valoir d'être transcrites sur les supports les plus officiels et pérennes, c'est-à-dire des inscriptions monumentales, sur l'autel lui-même ou un mur proche. Certaines comprennent des listes de reliques. A Rome, les plus anciennes inscriptions de consécration sur pierre incluant des listes de reliques remontent au $\mathrm{VII}^{\mathrm{e}}$ siècle. Certaines, aux VII ${ }^{\mathrm{e}}-\mathrm{VIII}^{\mathrm{e}}$ siècles, semblent avoir été composés directement pour le marbre et ne pas reprendre des textes sur parchemin ou papyrus. Les plus nombreuses datent cependant des $\mathrm{XII}^{\mathrm{e}}$ et $\mathrm{XIII}^{\mathrm{e}}$ siècle $^{88}$. L'Espagne a conservé elle aussi des inscriptions précoces, notamment à partir du VII ${ }^{\mathrm{e}}$ siècle $^{89}$. Sur 274 inscriptions de consécrations d'autels et d'églises du VIII ${ }^{\mathrm{e}}$ au XIII ${ }^{\mathrm{e}}$ siècle connues sur l'actuel territoire français, seules 34 soit $12 \%$ précisent les reliques encloses, qui sont moins de six en moyenne $e^{90}$.

Les inscriptions conservées en Allemagne sont rares. Il y en a une exceptionnelle au monastère bénédictin Saint-Georges de Prüfening à l'ouest de Ratisbonne en Bavière, sur un panneau de terre cuite fixé au pilier sud-ouest de la croisée du transept (fig. I/2). Elle est datée de 1119 , dix ans après la fondation ${ }^{91}$. Trente reliques sont indiquées dans l'ordre hiérarchique. Un texte très proche, incluant la même liste de reliques, se trouve dans le livre

${ }^{87}$ Cf. note 54.

${ }^{88}$ Cf. la synthèse de Nine Robijntje MIEDEMA, Die römischen Kirchen im Spätmittelalter nach den Indulgentiae ecclesiarum urbis Romae', Tübingen, Max Niemeyer, 2001 (Bibliothek des Deutschen Historischen Instituts in Rom 97), p. 320. Cf. Julius VON PFLUCK-HARTTUNG, Papsturkunden auf Marmor, in Quellen und Forschungen aus italienischen Arbiven und Bibliotheken, 4, 1902, p. 167-183; Vincenzo FOCELLA, Iscrizioni delle chiese e d'altri edificii di Roma del secolo XI fino ai giorni nostri, 14 t., Rome, 1869-1884, et par Angelo SILVAGNI, Monvmenta epigraphica christiana saecvlo XIII antiqviora qvae in Italia finibvs adhvc exstant, t. 1, Vatican, 1943 (45 planches sans transcription ni commentaire).

89 Plusieurs exemples in BRAUN, Der christliche Altar, t. 1, p. 364-366; d'autres in Inscriptiones Hispaniae christianae, éd. par Ernst Willibald HÜBNER, Berlin, 1871-1900; Hildesheim/etc., Olms, 1975.

${ }^{90}$ Jean MICHAUD, Les inscriptions de consécrations d'autels et de dédicace d'églises en France du VIII au XIII' s. Epigraphie et liturgie, thèse de $3^{e}$ cycle inédite, Poitiers, Centre d'études supérieures de Civilisation médiévale, 1978. Les aspects relevant des reliques ont fait l'objet d'un article: Jean MICHAUD, Culte des reliques et épigraphie. L'exemple des dédicaces et des consécrations d'autels, in Edina BOZOKY, Anne-Marie HELVETIUS (éd.), Les reliques. Objets, cultes, symboles, actes du colloque international de l'université du Littoral-Côte d'Opale (Boulogne-sur-Mer), 4-6 septembre 1997, Turnhout, Brepols, 1999, p. 199-212.

${ }_{91}^{1}$ Les lettres et les ornements constituant la bordure de ce panneau de $41 \mathrm{~cm}$ sur 26 ont été imprimées à l'aide de matrices sur des bandes de terre alternativement rouge et blanche, avant cuisson. Cf. Ratisbona Sacra. Das Bistum Regensburg im Mittelalter. Ausstellung anläßlich des 1250jäbrigen Jubiläums der kanonichen Errichtung des Bistums Regenburg durch Bonifatius 739-1989, Munich/Zürich, Schnell und Steiner, 1989, p. 134-135, et les remarques techniques de Otto HupP, Die Prüfeninger Weiheinschrift vom Jahre 1119, in Studien aus Kunst und Geschichte. Friedrich Schneider zum 70. Geburtstage gevidmet, Fribourg en Brisgau, Herder, 1906, p. 185-186. 
des traditions compilé au monastère dans la seconde moitié du XII ${ }^{\mathrm{e}}$ siècle $^{92}$. Ce qu'on peut encore lire de deux autres inscriptions peintes aux extrémités du transept, relatives à la consécration d'autres autels en 1125 , concorde également avec un texte copié dans le livre des traditions ${ }^{93}$ : la notice était reprise sur deux supports.

Certaines inscriptions étaient particulièrement précieuses ou monumentales. Lorsque la cathédrale de Hildesheim fut rénovée par l'évêque Hezilo et consacrée en 1061, une inscription en lettres d'or citant 83 reliques fut apposée au revers de l'autel majeur ${ }^{94}$. Egalement disparue, une autre inscription en lettres de cuivre doré se trouvait à l'entrée de l'église Saint-Etienne de Worms, dont elle rappelait la consécration en 1055 en indiquant la liste des reliques de l'autel ${ }^{95}$. Dans le chœur de l'église de l'abbaye cistercienne de Veruela, près de Saragosse en Aragon, une inscription de consécration de 1248 s'étale sur les quatre colonnes du rond-point, avec une liste de 20 reliques $^{96}$.

\section{Oralité, liturgie et dévotion privée}

Les noms des reliques ont joué dans le culte de celles-ci un rôle essentiel, jusqu'à acquérir parfois un pouvoir propre. Au milieu du $\mathrm{IV}^{\mathrm{e}}$ siècle en Afrique, avant que le terme reliquiae ne s'impose, les reliques étaient elles-mêmes désignées comme des "noms", nomen ou nomina martyrum ${ }^{97}$. Dans les Flores epytaphii sanctorum, traité où Thiofrid d'Echternach élabore au tournant des $\mathrm{XI}^{\mathrm{e}}-\mathrm{XII}^{\mathrm{e}}$ siècle la seule typologie de reliques que le Moyen Age ait laissé, figure dans la catégorie des restes non corporels un chapitre consacré aux noms des saints. Les noms prononcés des saints ont le même pouvoir que les reliques, et leur sont même supérieurs car mobilisables en tout lieu, au contraire des reliques dont la matérialité

${ }^{22}$ Cf. Hans Georg SCHMITZ, Kloster Prüfening im 12. Jabrbundert, Munich, Wöffle in Komm, 1975 (Miscellanea Bavarica Monacensia, 49), p. 31 pour la date et ici p. 44.

${ }^{93}$ On n'a plus retrouvé de l'inscription du mur sud, correspondant à un autel des apôtres, que des fragments illisibles, tandis que celle du mur nord (autel de la Vierge) est partiellement recouverte par un autel de 1712. Cf. Hans Karlinger, Georg Hager, Georg Lill, Die Kunstdenkmäler von Oberpfalq und Regensburg XX: Beqiksamt Stadtambof, Munich: Oldenbourg, 1914 (Die Kunstdenkmale des Königreiches Bayern, 176), p. 182, 188 et fig. 134 pour l'inscription de 1125 et p. 222 pour l'inscription de terre.

${ }^{94}$ Ed. Christine Wulf, Die Inschriften der Stadt Hildesheim, 2003 (Deutsche Inschriften LXVIII, Göttinger Reihe, 10), $\mathrm{n}^{\circ} 24$.

${ }^{95}$ Ed. Rüdiger Fuchs (éd.), Die Inscbriften der Stadt Worms, 1991 (Deutsche Inschriften XXIX, Mainzer Reihe, 2), $\mathrm{n}^{\circ} 10$.

${ }^{96}$ Robert FAVREAU, Epigraphie médiévale et hagiographie, in Le culte des saints, actes du colloque tenu à Poitiers les 15-17 septembre 1993, Poitiers, CESCM, 1995 (Civilisation médiévale, 1), p. 63-83; repris in ID., Etudes d'épigraphie médiévale, Limoges, 1995, t. II, p. 568-621, ici p. 77-78.

${ }_{77}$ Cf. Giuseppe Gagov, Il Termino "Nomina" sinonimo di "Reliquiae" nell'antica Epigrafia cristiana, in Miscellanea Francescana, 55, 1955, p. 3-13. 
limite l'effet à une unique zone sacralisée ${ }^{98}$. Thiofrid ne parle pas des noms écrits, mais il va de soi que les inventaires pouvaient servir de support à de telles pratiques de dévotion. A la fin d'un inventaire des reliques rédigé en 1003 à l'abbaye bénédictine de Prüm figure l'injonction "Lisez les noms des saints de manière à obtenir par leurs prières, la vie éternelle"" .

Certains inventaires étaient destinés à être lus en public. A la cathédrale d'Exeter, un inventaire transcrit vers 1020-1040 sur un cahier de huit feuilles, d'abord indépendant, a ensuite été relié avec un évangéliaire offert entre 1050 et 1072 . Recensant 138 reliques classées hiérarchiquement, dont il attribue le don au roi Aedelstan (924-940), il a été rédigé vers 1010 en ancien anglais et s'adresse dans son préambule à des auditeurs: "maintenant je veux vous dire sans faute ce qu'est le trésor de reliques (baligdom) qui est dans cette sainte cathédrale, et vous livrer des informations qui éclairent sans aucun doute ce que signifie chacune de ces reliques". Le texte explique de fait qui sont certains saints, ce que ne font pas les inventaires purs ${ }^{100}$.

Chaque 30 septembre à la Sainte-Chapelle de Paris, on commémorait l'arrivée d'un ensemble de reliques, de la Passion en particulier, que le roi Louis IX avait acquises auprès de l'empereur latin de Constantinople entre 1239 et 1241 . Une dizaine de pièces en latin chantées lors de cette fête annuelle, appelées De Sanctis Reliquiis, figurent dans un livre de séquences confectionné probablement vers 1250-1260 pour la chapelle privée du roi. On y énumère une grosse vingtaine de reliques. Ce sont les mêmes qui apparaissent dans les textes historiques et administratifs, à savoir le récit de translation et l'acte de cession des reliques qui sont des années 1240, et quatre inventaires réalisés de 1534 à 1791 . Mais les séquences chantées ne sont pas de simples listes. Elles citent les reliques dans un ordre bien déterminé, ce qui les amène à dérouler le récit de la Passion, et surtout elles commentent les objets les uns après les autres, les rapportant à diverses vertus chrétiennes ou soulignant le thème de la royauté divine ${ }^{101}$. La forme et l'usage de l'inventaire permettent d'appuyer la royauté capétienne sur le récit fondateur chrétien, en instrumentalisant soigneusement la collection. Ceci rappelle, sur un mode beaucoup plus précis, les interférences observées

98 At quod fidenter dictum sit salua omnium sanctorum gratia, multo gloriosiora sunt eorum nomina quam semata, un peu plus loin: Ubique sunt fidei presentia, cum corpore longe sint absentia. THIOFRID D'ECHTERNACH, Flores epytaphii sanctorum, III, 1 (De sanctorum nominum gratia et potentia), éd. Michele Camillo Ferrari, 1996 (Corpus Christianorum. Continuatio Mediaevalis, 133), 1. 130-131, 1. 154 et introduction p. XXXI.

${ }^{99}$ Nomina sanctorum sic perlege scripta piorum, Horum quo precibus sit tibi summa salus. Cet inventaire connu par une copie du XVI e siècle est donné dans une éd. de 1750 et trad. par Auguste DigOT, Inventaire du trésor de l'abbaye de Prüm, in Bulletin monumental, 15, 1849, p. 283-300, ici p. 297. Ed. nouvelle mais partielle, sans ce passage: Mittelalterliche Schatzverzeichnisse (cf. note 43), $\mathrm{n}^{\circ} 74$.

100 Bodl. Auct. D. 2. 16, f. 8r-14r. Cf. Max FÖRSTER, Zur Geschichte des Reliquienteultus in Altengland, Munich, 1943 (Bayerische Akademie der Wissenschaften, phil.-hist. Abt. Sitzungsberichte 1943, 8), p. 24-114, éd. p. 63-80. Je traduis d'après la traduction allemande de Förster.

${ }^{101}$ Karen Gould, The Sequences De Sanctis Reliquiis as Sainte-Chapelle Inventories, in Mediaeval Studies, 43, 1981, p. 315-341. 
plus haut entre litanies, inventaires et Laudes Regiae, qui liaient déjà les prières, les collections et le pouvoir carolingien.

L'inventaire d'Exeter classe les reliques dans l'ordre hiérarchique, ce qui ne peut correspondre à une ostension de reliquaires. On ignore si les reliques de la Sainte-Chapelle y faisaient l'objet d'une présentation spéciale pendant le chant de ces textes, ce qui a fortiori est moins sûr encore dans la chapelle privée du roi. Les usages liturgiques d'autres inventaires suivent cependant de près les mouvements des reliques et des reliquaires. Posés sur les autels, portés en procession lors de fêtes diverses, les reliquaires mis en scène isolément ne posaient aucun problème d'identification tant qu'il s'agissait d'un saint patron ou du saint du jour. Il en allait autrement avec des rituels impliquant les reliques collectivement, c'est-à-dire en tant que collection. Il fallait alors, pour être intelligible, s'appuyer sur des inventaires servant de trame: ils étaient le seul moyen de superviser des séries un peu importantes.

Les grandes ostensions de reliques donnèrent donc lieu à la réalisation d'inventaires des reliques montrées. L'abbé de Saint-Pierre-le-Vif à Sens et le prieur de Saint-Loup-deNaud publièrent ensemble en 1455 , sur une charte de parchemin, l'inventaire d'une partie importante de leurs reliques et la description de plusieurs reliquaires qui seraient envoyés sur les routes pour rassembler les fonds nécessaires à la reconstruction de leurs églises ${ }^{102}$. Lorsque les ostensions n'étaient pas itinérantes, les inventaires étaient rédigés sur des supports divers ${ }^{103}$, notamment des ordines d'ostensions. Il faut en distinguer les documents, plus rarement conservés, qui étaient directement employés dans le rituel d'ostension par les vocalissimi. Du bout de leur baguette, ceux-ci présentaient les reliques à la foule amassée devant leur estrade. Ils utilisaient des rouleaux de parchemin qu'un bâton fixé à l'extrémité permet de tenir ou d'enrouler, comme celui qui fut réalisé à Nuremberg après 1437 et actualisé jusqu'en $1459^{104}$, ou alors des cahiers, tel celui figuré sur une gravure de 1487 montrant un moment de l'ostension de Nuremberg toujours ${ }^{105}$. Deux rouleaux de parchemin provenant de l'abbaye prémontrée Saint-Vincent de Wrocław, datés entre 1401 et 1404, comportent la même liste de 80 reliques et une bulle d'indulgence accordée pour une

${ }^{102}$ Ed. Julliot, Prou, Le livre des reliques, p. 261-268. Le même abbé de Sens fonda, toujours au profit des restaurations, une confrérie qui elle aussi met en avant la collection: elle était vouée à tous les saints dont le monastère conservait les reliques. Cf. H. BOUvIER, Histoire de l'abbaye Saint-Pierre-le-Vif de Sens, ex-trait du Bulletin de la Société des sciences de l'Yonne, 1891 (tiré à part, Auxerre, 1891), p. 150-151.

${ }_{103}$ Cf. Hartmut KÜHNE, Ostensio reliquiarum. Untersuchung über Entstebung, Ausbreitung, Gestalt und Funktion der Heiltumsweisungen im römisch-deutschen Regnum, Berlin/New York, De Gruyter, 2000, chap. "Liturgische Quellen" p. 51-55 et les chap. "Quellen und Zeugen" relatifs à chacune des ostensions.

${ }^{104}$ Ill. in Nürnberg. Kaiser und Reich, cat. exp., Nuremberg, Neustadt an der Aisch, Degener, 1986, n 57 , p. 68 sq.

${ }^{105}$ Ill. ibid., $\mathrm{n}^{\circ} 54$ et p. 61 . 
ostension $^{106}$. Ils sont dotés sur les petits côtés de baguettes de bois et l'écriture est de particulièrement grand module, ce qui laisse penser qu'ils étaient utilisés lors de l'ostension, par les vocalissimi ou pour les afficher aux yeux de tous.

Même des reliques enfermées dans les autels, et donc invisibles, pouvaient attirer des pèlerins si l'église était particulièrement réputée et que l'on en faisait la publicité. C'était le cas des églises romaines. De nombreuses inscriptions de consécration monumentales trouvèrent ainsi un écho supplémentaire dans les récits de pèlerinage, largement diffusés en plusieurs langues à la fin du Moyen Age, sur support manuscrit puis imprimé. Les descriptions d'églises intégraient des transcriptions d'inscriptions parfois inaccessibles aux laïcs, puisque situées dans les chevets ${ }^{107}$, faisant connaître aux voyageurs les reliques dont ils imagineraient la présence dans le cœur obscur des autels, avant de se remettre en mémoire l'objet de leur dévotion une fois revenus chez eux.

La reproduction massive de certains inventaires de reliques accompagnait naturellement cette extension vers la sphère privée du culte des reliques détenues par les institutions ecclésiastiques. Le texte des formulaires utilisés lors de certaines grandes ostensions de reliques de l'Empire germanique fut ainsi imprimé, à partir des années 1460 , sur des feuilles ou des petits cahiers destinés à la vente, souvent dotés de gravures sur bois figurant les reliquaires ${ }^{108}$.

\section{Philippe Cordez}

EHESS, Paris / Humboldt-Universität, Berlin.

\footnotetext{
${ }^{106}$ Les deux inventaires, mesurant $71 \mathrm{~cm}$ de long sur respectivement $4,9 \mathrm{~cm}$ et $6,6 \mathrm{~cm}$ de large, étaient conservés en 1926 sous la cote Rep. 67n. 1524.IV.1 au Staatsarchiv de Breslau, aujourd'hui Archiwum Państwowe we Wrocławiu. Cf. Leo SANTIFALLER, Quellen zur Geschichte des spätmittelalterlichen Ablaß- und Reliquienwesens aus schlesischen Archiven, in Mitteilungen des Österreichischen Staatsarchives, 1, 1948, p. 20-136, ici p. 102-106, avec éd.

${ }^{107}$ Cf. MiEdEMA, Die römischen Kirchen, notamment p. 327-328.

${ }^{108}$ Outre le livre cité de H. KÜHNE, cf. CORDEZ, Die Wallfahrt.
} 


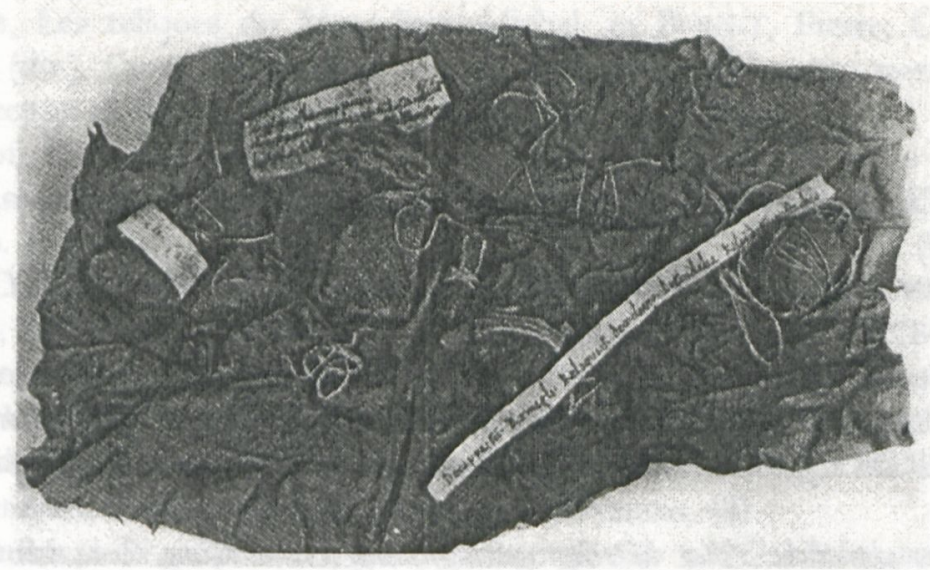

Figure I/1. Photo d'après Ph. GEORGE, Les reliques de Stavelot-Malmedy, 1989, p. 15.

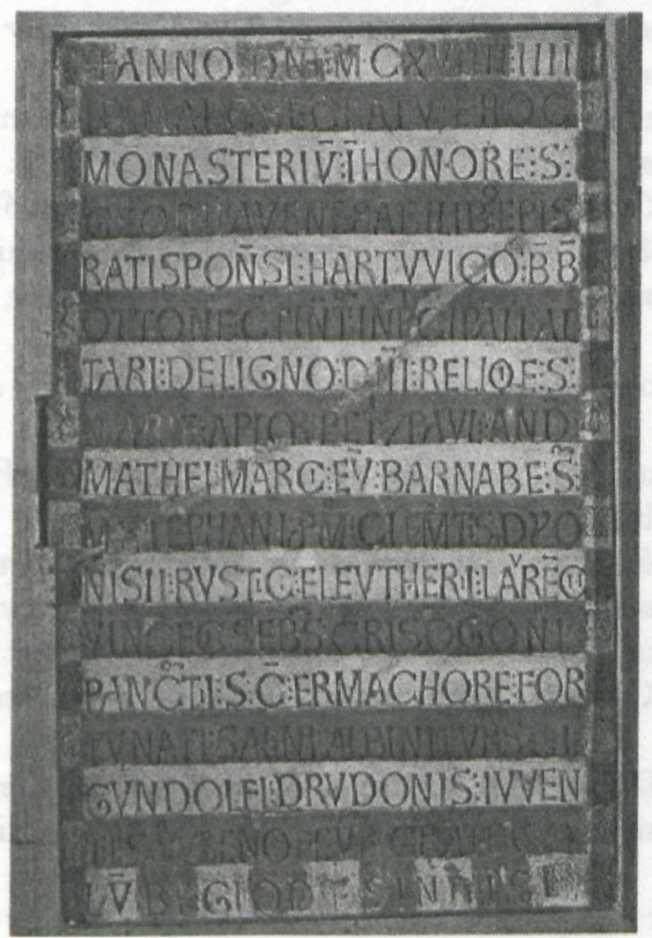

Figure I/2. Panneau du monastère de Saint-Georges de Prüfening. 


\section{Philippe CORDEZ}

\section{BIBLIOGRAPHIE}

ANDRIEU Michel, Le pontifical romain au Moyen Age, 4 t., Rome, Città del Vaticano, 1938-1941 (Studi et Testi, 86-88 et 99)

ANGILBERT DE SAINT-RIQUIER, De ecclesia Centulensi libellus, éd. G. Waitz, MGH SS 15,1, Hanovre, Hahn, 1887, p. 173-179.

BERTRAND Paul, Authentiques de reliques: authentiques ou reliques?, à paraître in Le Moyen Age.

BOOCKMANN Andrea, Die verlorenen Teile des "Welfenschatzes" Eine Übersicht anband des Reliquienverzeichnisses von 1482 der Stiftskirche St. Blasius in Braunschweig, Göttingen, Vandenhoeck \& Ruprecht, 1997

BRAUN Joseph, Der christliche Altar in seiner geschichtlichen Entwicklung, 2 t., Munich, Alte Meister Guenther Koch \& Co., 1924.

Chartae latinae antiquiores. Facsimile-Edition of the Latin Charters prior to the Ninth Century, t. 18, France V1, éd. Hartmut Atsma, Pierre Gasnault, Robert Marichal, Jean VeZIN, Dietikon/Zurich, Graf, 1985 et t. 19, France VII, éd. Hartmut ATSMA, Robert MARICHAL, Jean VEZIN, Dietiekon/Zurich, Graf, 1987.

CORDEZ Philippe, Die Wallfahrt als Medienereignis. Korpus und Deutungsversuche der Heiltumsverzeichnisse mit Reliquienbildern im Heiligen Römischen Reich (1460-1520), in TACKE, Andreas (dir.), "Ich armer sundiger Mensch". Heiligen- und Reliquienkult in der Zeitenwende Mitteldeutschlands, Göttingen, Wallenstein (à paraître en 2006)

DuBOIS Jacques (Dom), Le trésor des reliques de l'abbaye du Mont-Saint-Michel, in Millénaire monastique du Mont-Saint-Michel, 1, Paris, P. Lethielleux, 1967, p. 501-593.

GEOFFroy DE COURLON, Chronique de l'abbaye de Saint-Pierre-le-Vif de Sens, éd. et trad. par Gustave JUlliot, Sens, Duchemin, 1876 (Publications de la société archéologique de Sens, 2).

GEORGE Philippe, Les reliques de Stavelot-Malmedy. Nouveaux documents, Malmédy, Art et Histoire, $1989, \mathrm{n}^{\circ} 43$.

HERRMANN-MASCARD Nicole, Les reliques des saints: formation coutumière d'un droit, Paris, Klincksieck, 1975.

JULliOT Gustave, PROU Maurice, Le livre des reliques de l'abbaye Saint-Pierre-le-Vif de Sens publié avec plusieurs appendices, Sens, Duchemin, 1887.

KÜHNE Hartmut, Ostensio reliquiarum. Untersuchung über Entstebung, Ausbreitung, Gestalt und Funktion der Heiltumsweisungen im römisch-deutschen Regnum, Berlin/New York, De Gruyter, 2000.

Mittelalterliche Schatzverzeichnisse, t. 1, Von der Zeit Karls des Großen bis zur Mitte des 13. Jahrhunderts, éd. par le Zentralinstitut für Kunstgeschichte en collaboration avec Bernhard BISCHOFF, Munich, Prestel-Verlag, 1967.

MIEDEMA Nine Robijntje, Die römischen Kirchen im Spätmittelalter nach den Indulgentiae ecclesiarum urbis Romae', Tübingen, Max Niemeyer, 2001 (Bibliothek des Deutschen Historischen Instituts in Rom 97)

MOSLER Hans, Das Camper Reliquienverzeichnis von 1472, in Annalen des Historischen Vereins für den Niederrbein, insbesondere das alte Erzbistum Köln, 168/169, 1967, p. 60-101. 
Neveux François, Les reliques du Mont-Saint-Michel, in Bouet, Pierre, OTRANTO, Giorgio, VAuChez, André (dir.), Culte et pelerinages à Saint Michel en Occident. Les trois monts dédiés à l'Archange, Rome, 2003 (Collection de l'École française de Rome, 316), p. 245-269.

POMinN Krzysztof, Pour une histoire des sémiophores. A propos des vases des Médicis, 1986, repris sous le seul sous-titre in Des saintes reliques à l'art moderne. Venise-Chicago. XIII-XX' siècle, Paris, Gallimard, 2003, p. 147-161.

PROU Maurice, Chartraire Eugène, Authentiques de reliques conservées au Trésor de la cathédrale de Sens, in Mémoires de la Société nationale des Antiquaires de France, 59, 1890, p. 129-172.

Reudenbach Bruno et Gia Toussaint Gia, Die Wahrnehmung und Deutung von Heiligen. Überlegungen zur Medialität von Reliquiaren, in Das Mittelalter, 8, 2003, p. 34-40.

RÖCKELEIN Hedwig, Reliquientranslationen nach Sachsen. Über Kommunikation, Mobilität und Öffentlichkeit in Frühmittelalter, Sttugart, Thorbecke, 2002 (Beihefte der Francia, 48)

SPILLING Herrad, Sanctarum reliquiarum pignera gloriosa. Quellen zur Geschichte des Reliquienschatzes der Benediktinerabtei Zwiefalten, Bad Buchau, Federsee-Verlag, 1992.

STÜCKELBERG Ernst Alfred, Geschichte der Reliquien in der Schweiz, 2 t., Zurich, 1902-1908 (Verlag der Schweizerischen Gesellschaft für Volkskunde, 1 et 5) 